\title{
VEGETACIÓN DE LA CORDILLERA ANTEQUERANA ORIENTAL (SUBSECTOR TORCALENSE). MÁLAGA-GRANADA (ESPAÑA)
}

\author{
Andrés V. PÉREZ LATORRE*, Gonzalo CABALLERO, \\ Federico CASIMIRO-SORIGUER SOLANAS, Oscar GAVIRA y Baltasar CABEZUDO \\ Departamento de Biología Vegetal. Universidad de Málaga. Apdo. 59. 29080 Málaga. \\ *Autor para correspondencia: avperez@uma.es
}

Recibido el 30 de octubre de 2008, aceptado para su publicación el 15 de abril de 2009

Publicado "on line" en mayo de 2009

RESUMEN. Vegetación de la cordillera Antequerana Oriental (subsector Torcalense). Málaga-Granada (España). Se ha realizado un estudio fitocenológico, florístico y fitogeográfico del subsector Torcalense en su parte sudoriental (sector Antequerano, provincia Bética, Región Mediterránea), que abarca diversas sierras del Arco Calizo Central de Málaga y que se adentran en Granada (Andalucía, España). Bioclimáticamente aparecen los pisos termomediterráneo subhúmedo, mesomediterráneo subhúmedo-húmedo y supramediterráneo húmedo. Se propone una sectorización fitogeográfica a un nivel inferior al de sector, con 2 unidades: unidad Pedrizas (la más occidental), y unidad de Zafarraya (más oriental). Se han seleccionado algunos táxones de interés por su grado de amenaza (ej. Salvia candelabrum), por estar protegidos (ej. Prunus mahaleb) o por ser endemismos torcalenses (Saxifraga biternata) o muy escasos (ej. Narcissus elegans). El territorio presenta una elevada cantidad de sintaxones (49) si tenemos en cuenta su pequeña extensión y homogeneidad litológica. De estos sintaxones destacan algunas novedades como un nuevo tipo de quejigal (Vinco difformis-Quercetum fagineae). Se han catalogado 8 series de vegetación: 4 de tipo climatófilo caracterizadas por vegetación climácica de árboles planifolios, perennifolios o marcescentes (Quercus rotundifolia, Quercus faginea) 1 edafoxerófila con acebuches (Olea sylvestris) y 3 edafohidrófilas con vegetación climácica dominada por árboles caducifolios (Ulmus minor, Fraxinus angustifolia) o arbustos (Nerium oleander) de riberas o niveles freáticos. La vegetación de complejos edafogénicos se desarrolla en karst (Acer monspessulanum) o lagunas (Ranunculus saniculifolius, $R$. tricophyllus). Se aportan en este trabajo las especies de interés, el esquema sintaxonómico, los inventarios y/o tablas fitosociológicas de novedades o interés especial y un mapa fitogeográfico.

Palabras clave. Vegetación, fitogeografía, sintaxonomía, sector Antequerano, Málaga, Andalucía, España.

ABSTRACT. Vegetation of the eastern Antequera mountain range (Torcalense subsector). Malaga-Granada (Spain). We have studied the vegetation and flora of the south-eastern Torcalense phytogeographical subsector (Antequerano sector, Bética province, Mediterranean region). This area comprises several mountain ranges in the centre of the province of Malaga, entering western Granada province. Three bioclimatic belts have

Proyecto financiado por la Consejería de Medio Ambiente de la Junta de Andalucía (España) (Contrato 8.06/03.2434 UMA, NET090897/1 EGMASA) 
been detected: thermomediterranean subhumid, mesomediterranean subhumid-humid and supramediterranean humid. Two phytogeographical units, under subsector level, are proposed: Pedrizas unit (western) and Zafarraya unit (eastern). Some interesting taxa have been found in the territory; they are endangered (e. g. Salvia candelabrum), protected (e. g. Prunus mahaleb), local endemism (Saxifraga biternata) or scarce plant (e. g. Narcissus elegans). The studied area shows a high number of plant communities (49) if we take into account its little area and homogeneous lithology. We point out some new syntaxa as a Quercus faginea forest (Vinco difformis-Quercetum fagineae). A total of 8 vegetation series have been detected: 4 series are climatophyllous and characterised by sclerophyllous or winter-deciduous broad-leafed trees (Quercus rotundifolia, Quercus faginea); 1 edaphoxerophyllic series is characterised by Olea sylvestris; 3 series are edaphohidrophyllic and characterised by deciduous trees (Ulmus minor, Fraxinus angustifolia) or tall shrubs (Nerium oleander) in riversides and river beds with underground water tables. The vegetation that develops as mosaic-complexes belongs to karst (Acer monspessulanum) or pools (Ranunculus saniculifolius, $R$. tricophyllus). The more interesting species, a syntaxonomical scheme, relevés and tables, and a phytogeographical map are included.

Key words. Vegetation, Phytogeography, syntaxonomy, Antequerano sector, Malaga, Andalusia, Spain.

\section{INTRODUCCIÓN Y OBJETIVOS}

En el marco de los trabajos que se están realizando sobre cartografia de la vegetación a escala 1:10.000 de la Comunidad Autónoma de Andalucía, hemos cartografiado e inventariado la flora y vegetación de un conjunto de sierras (Cordillera Antequerana, Dorsal Bética o Arco Calizo Central de Málaga) de gran importancia fitogeográfica por su función de conectividad entre las serranías Bético-orientales y la Serranía de Ronda (Bético-occidental). Este estudio se ha centrado en su parte más oriental, que se incluye al igual que el resto en el subsector Torcalense (sector Antequerano, provincia Bética, región Mediterránea) (fig. 1).

El subsector fitogeográfico Torcalense se encuentra relativamente conservado debido a su litología calizo-dolomítica con suelos poco desarrollados lo que ha impedido su aprovechamiento agrícola, aunque no así el ganadero. Son frecuentes las zonas donde aún se pueden encontrar representaciones de la vegetación natural, localizándose las representaciones más interesantes de flora y vegetación en las zonas más rocosas.
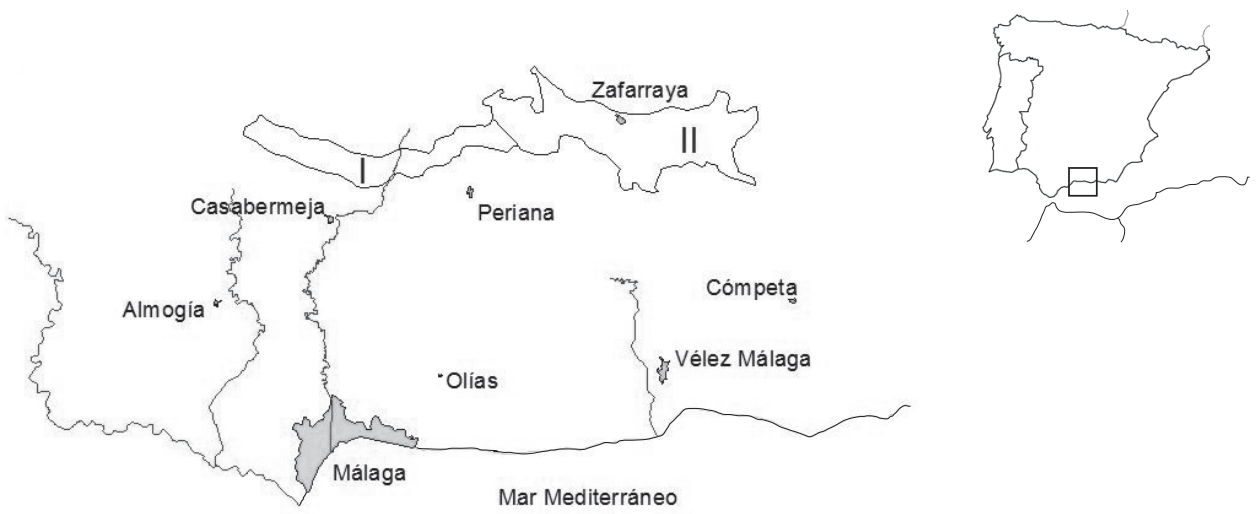

Figura 1. Localización y unidades fitogeográficas del territorio estudiado. I: Pedrizas, II: Zafarraya. Study area and phytogeographical units. 
Los estudios botánicos en el subsector Torcalense se han centrado básicamente en el Torcal de Antequera (Ceballos y Vicioso, 1933; Guerra, 1982; Guerra et al., 1988; Asensi et al., 2005), un lugar clásico para la Botánica española, o en el Boquete y Poljé de Zafarraya (Nieto, 1987) de gran interés, dejando de lado el resto de sierras de la unidad, que presentan sin embargo una gran fitodiversidad.

En este trabajo se ha inventariado la vegetación durante los años 2006 y 2007, lo que nos ha permitido detectar la existencia de numerosas especies y comunidades no citadas previamente en este subsector y que aumentan su importancia fitogeográfica.

Los objetivos del presente trabajo, independientemente de la cartografía en detalle levantada, han sido la realización de un catálogo florístico básico (con cerca de 500 táxones), del que solo presentamos las especies de interés, y sobre todo el estudio de las comunidades vegetales (sinecología, composición florística y esquema sintaxonómico), centrándonos en las novedades sintaxonómicas (tablas de asociación/comunidad), las series de vegetación y complejos edafogénicos y su dinamismo. En base a los fitoindicadores se propone una sectorización bioclimática y fitogeográfica del territorio.

\section{MATERIAL Y MÉTODOS}

\section{Área de estudio}

Ocupa territorios en las provincias de Málaga y Granada y se corresponde (fig. 1) con la parte más oriental del subsector Torcalense (Pérez Latorre \& Cabezudo, 2002) en la llamada geográficamente "Dorsal Bética" (Yus et al., 2007) o Cordillera Antequerana (Pérez Latorre \& Cabezudo op. cit.). Los límites van de oeste a este del siguiente modo: en Málaga la base del Torcal de Antequera y los piedemontes de las Sierras de las Cabras, Las Pedrizas y Camorolos (LIC de Camorolos) incluyendo desde ahí la Sierra del Rey, La Sierra de Vilo y Enmedio, los Tajos del Fraile y Doña Ana y Gomer y Sierra de la Torca; ya en Granada las Sierras del Boquete de Zafarraya y Sierras de Júrtiga y La Torrecilla en las cercanías de Alhama de Granada.

\section{Metodología}

El estudio bioclimático se ha basado en los conceptos de piso bioclimático y ombrotipo (Rivas Martínez, 1987), utilizando los datos proporcionados por el Instituto Nacional de Meteorología de estaciones en el territorio de estudio y alrededores. La inexistencia de estaciones por encima de $1000 \mathrm{~m}$ hace difícil la descripción bioclimática del territorio en el horizonte superior del piso mesomediterráneo y en el supramediterráneo, lo que se ha intentado subsanar usando fitoindicadores.

Para la sectorización fitogeográfica hemos tenido en cuenta las propuestas de Rivas Martínez (1988), Nieto Caldera et al. (1991, 1998), Rivas Martínez et al. (1997) y Galán de Mera et al. (2003), aunque la sectorización de referencia procede de Pérez Latorre \& Cabezudo (2002). Además los sectores se han dividido en unidades menores con referencia en principio sólo al área de estudio (unidades fitogeográficas). Para reconocer las distintas unidades fitogeográficas se han tenido en cuenta tanto bioindicadores florísticos como comunidades, series de vegetación, paisaje vegetal, bioclimatología y uso del territorio.

La flora vascular herborizada se conserva en el herbario MGC y para su nomenclatura e identificación se han seguido básicamente las obras "Flora Vascular de Andalucía Occidental" (Valdés et al., 1987), "Flora Iberica" (Castroviejo, 1986-2008) y "Catalogue des Plantes Vasculaires du Nord du Maroc" (Valdés et al., 2002) así como monografías de algunos géneros y familias (Poaceae, Carex).

Para la descripción de las unidades de vegetación se ha seguido el método fitosociológico de Braun-Blanquet (1979) con 


\begin{tabular}{llll}
\hline Localidad & It & P (mm) & piso / ombrotipo \\
\hline $\begin{array}{l}\text { Unidad Pedrizas } \\
\text { El Torcal }(1218 \mathrm{~m})\end{array}$ & 221 & 784 & mesomediterráneo superior, subhúmedo inferior \\
Unidad Zafarraya & & & \\
Alfarnate $(925 \mathrm{~m})$ & 262 & 1013 & mesomediterráneo medio, húmedo inferior \\
Alcaucín $(508 \mathrm{~m})$ & $\mathrm{T}=15,7$ & 989 & mesomediterráneo inferior, subhúmedo superior \\
Alcaicería $(780 \mathrm{~m})$ & $\mathrm{T}=14,3$ & 876 & mesomediterráneo medio, subhúmedo superior \\
\hline
\end{tabular}

Tabla 1. Datos bioclimáticos del territorio según unidad fitogeográfica $(\mathrm{T}=$ temperatura media anual en ausencia de It). Bioclimatic data in the studied area, distributed according to phytogeographical units ( $T=$ average annual temperature in absence of $I t)$.

las matizaciones aportadas por Schuhwerk (1990) y Gehú \& Rivas Martínez (1981), realizándose cerca de 120 inventarios distribuidos homogéneamente por el territorio. La vegetación ha sufrido antropización en parte, lo que da lugar a comunidades fragmentarias, descritas como comunidades basales (BC), derivadas (DC) y marginales (MC) (Foucault, 1981; Dierschke, 1993 y Kopecký et al., 1995). Para las nuevas propuestas nomenclaturales sintaxonómicas se ha consultado el Código Internacional de Nomenclatura Fitosociológica (CNF - Weber et al., 2000). Los diferentes estadíos dinámicos de la vegetación han sido analizados, en general, según la metodología sinfitosociológica (Rivas Martínez, 1987) con las propuestas tipológicas de Vigo (1998) y de Pérez Latorre et al. $(2004,2008)$ respecto a la zonopotencialidad de los territorios y teselas, la sinfenosucesión y las criptoseries.

\section{RESULTADOS Y DISCUSIÓN}

\section{Bioclimatología}

En la cordillera objeto de estudio predomina el piso mesomediterráneo aunque los fitoindicadores muestran la existencia del supramediterráneo a partir de unos $1300 \mathrm{~m}$ y del termomediterráneo en la vertiente sur hasta unos 700-800 m aunque, de modo topográfico, puede ascender un poco más. Es muy destacable el efecto solana-umbría en estas sierras debido a su orientación oeste-este, lo que hace que a una misma altitud podamos encontrarnos un piso termomediterráneo topográfico en orientación sur y un mesomediterráneo medio muy continentalizado en la cara norte. La geomorfología de navas y poljés también acrecienta el efecto de inversión térmica, pues se encuentran fitoindicadores supramediterráneos en estas condiciones (Berberis hispanica, Rhamnus saxatilis). Respecto al ombroclima, las precipitaciones van aumentando de oeste a este: el Torcal a pesar de sus $1200 \mathrm{~m}$ solo tiene ombrotipo subhúmedo, en la zona central (alrededores del Puerto de Los Alazores y Alfarnate a 900 m.) el ombrotipo es húmedo y luego las precipitaciones decrecen hacia el oriente.

\section{Fitogeografía}

El área de estudio (fig. 1) se encuadra en su totalidad en el sector Antequerano, ya formulado por Nieto et al. (1991) (en este caso como subsector Antequerano dentro del sector Hispalense) y modificado por Pérez Latorre \& Cabezudo (2002). El sector Antequerano se extiende por la zona central de Andalucía en las provincias de Málaga, Cádiz, Córdoba, Sevilla y Granada, con dos subsectores: Antequerano y Torcalense. El subsector Antequerano ocupa la altiplanicie antequerana y sus sierras-isla y los sustratos margo-yesíferos, siendo sus límites 


\begin{tabular}{lll}
\hline Sintaxones & sector Antequerano & sector Rondeño \\
\hline Matorral calcícola & $\begin{array}{l}\text { Genisto speciosae-Ulicetum } \\
\text { parviflorii / Genisto umbellatae- } \\
\text { Chronanthetum biflori }\end{array}$ & Cytiso plumosi-Ulicetum baetici \\
\hline Matorral dolomitícola & $\begin{array}{l}\text { Thymo gracilis-Lavanduletum } \\
\text { lanatae / Odontito purpureae- } \\
\text { Thymetum baetici } \\
\text { lavanduletosum lanatae }\end{array}$ & Lavandulo lanatae-Ulicetum baetici \\
\hline $\begin{array}{l}\text { Rupícolas meso / } \\
\text { supramediterráneas }\end{array}$ & $\begin{array}{l}\text { Linario anticariae-Saxifragetum } \\
\text { biternatae / Saxifragetum camposii }\end{array}$ & $\begin{array}{l}\text { Saxifragetum bourgaeanae / } \\
\text { Rhamno pumili-Saxifragetum } \\
\text { granatensis }\end{array}$ \\
\hline $\begin{array}{l}\text { Especies bioindicadoras } \\
\text { recuentes en los matorrales }\end{array}$ & $\begin{array}{l}\text { Ulex parviflorus } \\
\text { Cytisus fontanesii subsp. fontanesii }\end{array}$ & $\begin{array}{l}\text { Ulex baeticus s. l. } \\
\text { Cytisus fontanesii subsp. plumosus }\end{array}$ \\
\hline
\end{tabular}

Tabla 2. Algunas diferencias clave entre los sectores Antequerano y Rondeño: matorrales, rupícolas y fitoindicadores territoriales. Some key differences between Antequerano and Rondeño sectors: shrublands, rupicolous communities and phytogeographical plant indicators.

Coronil (al oeste), la Vega de Granada (al este), las subbéticas sevillanas y Cordobesas (al norte) y la cordillera del Torcal (al sur). El subsector Torcalense está formado por sierras calizas muy karstificadas que van desde El Chorro en Málaga (al oeste) hasta Zafarraya en Granada (al este) y la Sierra de Loja (nordeste); al sur limita con el Corredor de Colmenar (Málaga).

Otros autores (Rivas Martínez et al., 1997, Rivas Martínez et al., 2002) vienen proponiendo que la Cordillera del Torcal y la Sierra de Loja pertenecen al sector Rondeño. Sin embargo, no existen endemismos rondeños en la cordillera del Torcal ni en el resto de la unidad Antequerana, que tiene sus propios endemismos, como: Hippocrepis taveramendozae, Lepidium calycotrichum subsp. anticarium, Scrophularia viciosoi, Saxifraga biternata, Nepeta ametisthyna subsp. anticaria y Cytisus malacitanus subsp. moleroi. Existen además grandes diferencias fitosociológicas a nivel de sintaxones vicariantes, sobre todo matorrales y rupícolas (tabla 2).

El subsector Torcalense, que abarca desde la Sierra de Huma (Valle del Abdalajís, Málaga) hasta más allá del Boquete de Zafarraya y Sierra de Loja (Granada), se comporta como un corredor fitogeográfico para especies orófilas, calcícolas y dolomitícolas entre la Bética occidental y la oriental, pues al sur contacta con territorios de litología silíceo-margosa y al norte con territorios margo-yesosos, poco aptos para dicho contingente florístico. Estudios más detallados en esta zona de encrucijada fitogeográfica, como el realizado por Gavira (2006) para otro corredor en la Serranía de Ronda, pondrían aclarar finalmente la sectorización de estos territorios.

La zona de estudio (fig. 1) la encuadramos en el siguiente esquema fitogeográfico:

Reino Holártico

Región Mediterránea

Subregión Mediterránea - Occidental

Superprovincia Iberomarroquí - Atlántica Provincia Bética

Sector Antequerano

Subsector Torcalense

I. Unidad Pedrizas

II. Unidad de Zafarraya

Sector Antequerano, subsector Torcalense 
[Sector Rondeño, Distrito Anticariense sensu Rivas Martínez et al. (2002)]

El territorio estudiado se incluye por completo en este subsector y se extiende desde la base del Torcal (Málaga) hasta la Sierra de Alhama (Granada) pasando por el Puerto de las Pedrizas y las sierras del Boquete de Zafarraya. Se pueden distinguir dos unidades fitogeográficas:

I. Unidad Pedrizas: desde la base del Torcal hasta Los Alazores. La unidad es una cordillera caliza que en el territorio estudiado solo alcanza algunos isleos al sur de las sierras de Camorolos (Sierra del Rey), rodeados de navas con suelos arcillosos. El piso es mesomediterráneo subhúmedo y la serie dominante es la del Paeonio-Querceto rotundifoliae $S$, aunque son frecuentes los quejigales de Vinco-Querceto fagineae S. En la solana son característicos los acebuchales subrupícolas de Tamo-Oleeto sylvestris $S$. El matorral es de Genisto-Ulicetum parviflorii. En esta unidad se encuentran las representaciones más orientales conocidas de Saxifraga biternata.

II. Unidad de Zafarraya: desde Los Alazores y Puerto del Sol hasta la Sierra del Boquete de Zafarraya. También se trata de una cordillera caliza, pero con zonas dolomíticas, muy afectada por procesos de karstificación y con pisos mesomediterráneo y supramediterráneo ambos con ombrotipo húmedo (subhúmedo hacia el este). La serie dominante en las solanas son acebuchales de Tamo-Oleeto sylvestris $S$, en los llanos los encinares de PaeonioQuerceto rotundifoliae $S$, en las cumbres la serie es del Berberido-Querceto rotundifoliae $S$ y en las umbrías y navas los quejigales de Vinco-Querceto fagineae $S$. Llegan aquí matorrales dolomitícolas, indicadores béticoorientales y a la vez de continentalidad de Thymo-Lavanduletum lanatae y de termicidad de Odontito-Thymetum baetici. El Poljé de Zafarraya es un hito de primer orden en esta unidad, con restos de vegetación hidrófita.

Las características de esta cordillera, de gran longitud y poca anchura, alargada de oeste a este, y con singulares discontinuidades geográficas, provocan una significativa compartimentación fitogeográfica. Es posible que el subsector Torcalense en su totalidad (Pérez Latorre \& Cabezudo, 2002) pudiese ser dividido de modo provisional en 3 ó 4 unidades a él subordinadas: unidad de Abdalajís (Sierras de Huma y del Valle de Abdalajís), unidad del Torcal -incluiría la unidad Pedrizas estudiada en este trabajo- (Sierra Pelada, Torcal, Sierra de las Cabras, Las Pedrizas, Los Camorolos, Sierra de Gibalto) y unidad de Zafarraya (Sierras de Alfarnate, Zafarraya y Alhama). La Sierra de Loja, por sus especiales características geográficas, geomorfológicas y extensión, podría tener su propia unidad (Loja).

\section{Flora de interés}

A continuación presentamos un listado de las especies de cormófitos que consideramos más importantes debido a su grado de amenaza, por estar protegidas y/o ser endemismos locales o de las cordilleras béticas y muy escasas en el territorio. La secuencia de datos sobre cada taxón es: nombre y autores, biotipo, distribución general, distribución local, comportamiento ecológico, sintaxones principales en que aparece, categorías de amenaza (LRA=Lista Roja de la Flora Vascular Amenazada de Andalucía/ LFF=Ley 8/2003 de Flora y Fauna Silvestres de Andalucía) y/o rareza.

\section{Acer monspessulanum $\mathrm{L}$.}

Microfanerófito. Europa y Norte de África. Pedrizas, Zafarraya. Acerales. Comunidad de Acer monspessulanum y Pistacia terebinthus. NT (LRA); IE (LFF).

Armeria villosa subsp. longiaristata (Boiss. \& Reuter) Nieto Feliner

Hemicriptófito. Endemismo bético. Zafarraya. Rupícola. Biscutello-Saxifragetum reuteranae. Muy escasa en el territorio.

Centaurea clementei Boiss. ex DC. 
Caméfito. S de España y $\mathrm{N}$ de Marruecos. Pedrizas. Rupícola. Linario-Centaureetum clementei. VU (LRA)

Centaurea prolongoi Boiss. ex DC.

Hemicriptófito. Endemismo andaluz (bético). Pedrizas, Zafarraya. Rupícola. BiscutelloSaxifragetum reuteranae. VU (LRA).

\section{Cotoneaster granatensis Boiss.}

Microfanerófito. S y SE peninsular. Zafarraya. Espinares caducifolios. Lonicero-Berberidion hispanicae. NT (LRA).

\section{Crataegus granatensis Boiss.}

Microfanerófito. SE de la Península Ibérica y N de África. Zafarraya. Espinares caducifolios. Comunidad de Crataegus monogyna. Muy escaso en el territorio.

Cytisus arboreus subsp. catalaunicus (Web.) Maire

Microfanerófito. W de la Región Mediterránea. Pedrizas, Zafarraya. Zarzales, coscojares y bosques. EN (LRA)

Cytisus scoparius subsp. reverchonii (Degen \& Hervier) Rivas Goday \& Rivas Mart.

Nanofanerófito. Bético. Zafarraya. Escobonales. Genisto-Cytisetum reverchonii. Muy escasa en el territorio.

Delphinium emarginatum subsp. nevadense ( $\mathrm{G}$. Kunze) C. Blanché \& Molero

Criptófito. Endemismo andaluz (bético). Zafarraya. Quejigales. Vinco-Quercetum fagineae. VU (LRA)

\section{Dictamnus albus L.}

Caméfito. Región Mediterránea. Zafarraya. Matorrales. Odontito-Thymetum lavanduletosum. Muy escaso en el territorio.

\section{Hesperis laciniata All.}

Hemicriptófito. S de Europa y Marruecos. Pedrizas, Zafarraya. Rupícola. BiscutelloSaxifragetum reuteranae. Muy escaso en el territorio.

\section{Hippocrepis rupestris Laza}

Caméfito. Suribérico y Marruecos. Zafarraya. Rupícola. Biscutello-Saxifragetum reuteranae. Muy escaso en el territorio.

\section{Lavatera olbia L.}

Nanofanerófito. Mediterráneo Occidental. Pedrizas. Espinares. Spartio-Rubetum ulmifolii. Muy escasa en el territorio.

Narcissus elegans (Haworth) Spach

Criptófito. Mediterráneo Occidental. Pedrizas. Prados. Ranunculion bullati. Muy escasa en el territorio (única localidad ibérica).

Polygonatum odoratum (Miller) Druce

Criptófito. Europa, N de África y NW de Asia. Zafarraya. Encinares con quejigos. PaeonioQuercetum rotundifoliae quercetosum fagineae. Muy escaso en el territorio.

\section{Prunus insititia L.}

Nanofanerófito. S de Europa, $\mathrm{N}$ de África y SW de Asia. Pedrizas. Espinares. Com. de Crataegus monogyna. VU (LFF).

\section{Prunus mahaleb L.}

Nanofanerófito. C y S de Europa, W de Asia y NW de África. Zafarraya. Subrupícola. LoniceroBerberidion hispanicae. VU (LFF).

Ranunculus peltatus Schrank subsp. saniculifolius (Viv.) C. D. K. Cook

Hidrófito. Mediterráneo y Macaronésico. Zafarraya. Charcas temporales. CallitrichoRanunculetum saniculifolii. Muy escasa en el territorio.

\section{Ranunculus tricophyllus Chaix}

Hidrófito. Holártico. Zafarraya. Ríos. Ranunculion aquatilis. Muy escasa en el territorio.

\section{Quercus x senneniana A. Camus}

Mesofanerófito. Ibérico. Encinares con quejigos. Paeonio-Quercetum rotundifoliae quercetosum fagineae. Muy escasa en el territorio.

\section{Salvia candelabrum Boiss.}

Caméfito. Endemismo andaluz (bético). Zafarraya. Matorrales. Odontito-Thymetum baetici. VU (LRA). 
Tabla 3

\begin{tabular}{|c|c|c|c|c|c|c|c|c|c|c|}
\hline \multicolumn{11}{|c|}{$\begin{array}{l}\text { Vinco difformis-Quercetum fagineae Pérez Latorre \& Cabezudo ass. nova (5) } \\
\text { (Querco-Fagetea, Quercetalia pubescentis-petraeae, Aceri-Quercion fagineae) }\end{array}$} \\
\hline INVENTARIO N ${ }^{\circ}$ & 1 & 2 & 3 & 4 & 5 & 6 & 7 & 8 & 9 & 10 \\
\hline Orientación & $\mathrm{N}$ & $\mathrm{E}$ & $\mathrm{N}$ & - & NW & $\mathrm{N}$ & $\mathrm{NE}$ & $\mathrm{N}$ & $\mathrm{N}$ & NW \\
\hline Inclinación $\left({ }^{\circ}\right)$ & 10 & 10 & 15 & - & 30 & 15 & 25 & 20 & 25 & 45 \\
\hline Área $\left(\mathrm{m}^{2}\right)$ & 200 & 200 & 250 & 100 & 100 & 150 & 200 & 200 & 150 & 250 \\
\hline Litología & $\mathrm{Cal}$ & $\mathrm{Cal}$ & $\mathrm{Cal} / \mathrm{Mar}$ & $\mathrm{Cal}$ & $\mathrm{Cal} / \mathrm{A}$ & $\mathrm{Cal} / \mathrm{Arc}$ & $\mathrm{Cal}$ & Cal & Cal & Mac \\
\hline Altitud (m) & 830 & 820 & 900 & 950 & 700 & 930 & 750 & 1100 & 1000 & 1200 \\
\hline Altura vegetación $(\mathrm{m})$ & 10 & 12 & 10 & 10 & 9 & 15 & 12 & 12 & 8 & 12 \\
\hline \multicolumn{11}{|c|}{ Características y diferenciales de asociación } \\
\hline Quercus faginea subsp. faginea & 4 & 5 & 5 & 5 & 5 & 3 & 4 & 4 & 4 & 5 \\
\hline Lonicera etrusca & - & - & + & 2 & - & + & - & 2 & + & + \\
\hline broteroi & - & - & + & - & - & + & + & 1 & + & 2 \\
\hline Vinca difformis & 2 & 3 & - & - & 1 & 1 & 2 & - & - & - \\
\hline Bryonia dioica & + & - & - & - & + & 1 & + & - & - & - \\
\hline Cytisus arboreus $c$ & 1 & - & + & - & - & - & + & 1 & - & - \\
\hline Ranunculus blepharicarpos & + & - & + & - & - & - & - & - & 1 & - \\
\hline \multicolumn{11}{|c|}{ Características de unidades superiores (Querco-Fagetea y Rhamno-Prunetea) } \\
\hline Crataegus monogyna & 2 & + & 3 & 2 & 2 & 3 & 2 & 3 & + & 1 \\
\hline Tamu & 2 & 1 & + & 3 & 2 & 1 & + & 1 & 1 & + \\
\hline ium sylvaticum & - & 3 & 1 & - & 1 & 1 & + & 1 & 1 & 1 \\
\hline Rosa & - & - & 1 & 2 & 1 & + & 1 & - & + & - \\
\hline nсеит & - & - & + & - & 1 & + & 1 & - & 2 & - \\
\hline lius & - & 1 & + & 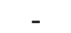 & - & + & - & 1 & 2 & - \\
\hline terebinthus & + & - & - & 2 & - & - & + & + & - & - \\
\hline Heder & - & + & - & - & - & 1 & - & 1 & 1 & - \\
\hline essulanu & - & + & - & - & - & - & - & 2 & + & - \\
\hline Helleborus foetidus & - & + & - & - & - & - & - & 1 & - & + \\
\hline Geum sylvaticum & _- & - & + & _ & - & _ & - & - & - & + \\
\hline
\end{tabular}

Otras características: en 2 Clematis vitalba 1 . En 3 Cephalanthera longifolia + . En 5 Rosa sempervirens +. En 6 Arum italicum +. En 7 Prunus insititia +. En 8 Cotoneaster granatensis +, Prunus spinosa 1, Rosa micrantha 2. En 12 Hedera hibernica 2.

Características de unidades superiores (Quercetalia y Pistacio-Rhamnetalia)

Rubia peregrina

Ruscus aculeatus

Asparagus acutifolius

Lonicera implexa

Quercus rotundifolia

Smilax aspera

Rhamnus alaternus

Aristolochia baetica

Carex hallerana

Clematis flammula

Osyris alba

$\begin{array}{lllllll}+ & 1 & 1 & - & 2 & + & - \\ 1 & 2 & - & 1 & - & + & 1 \\ + & - & + & - & + & + & 1 \\ + & 1 & 2 & - & 1 & - & - \\ + & + & + & + & - & - & - \\ - & 1 & - & - & 1 & + & + \\ - & - & 1 & + & + & - & - \\ - & - & - & - & - & + & + \\ - & - & 1 & - & - & - & + \\ - & - & + & - & - & - & - \\ - & - & - & - & 1 & - & 1\end{array}$

Otras características: en 3 Anemone palmata + . En 5 Pulicaria odora + , Teucrium fruticans 1.

\section{Compañeras}

Geranium purpureum

Geranium lucidum

Ulex parviflorus

Anacamptis pyramidalis

Dactylis glomerata hispanica

Phlomis purpurea

Aristolochia paucinervis

Bellis sylvestris

Cynosurus echinatus

Euphorbia characias

Geranium malviflorum

Himanthoglossum hircinum

Hyacinthoides hispanica

Muscari comosum

Ophrys lutea

Thapsia villosa

\begin{tabular}{llllllllll}
+ & 2 & - & 1 & 1 & 2 & 3 & + & - & 3 \\
+ & - & - & - & - & - & 1 & - & + & 3 \\
- & - & - & + & + & - & - & + & 2 & - \\
+ & - & - & - & - & - & - & + & - & + \\
1 & - & - & 1 & - & + & - & - & - & - \\
+ & - & - & - & + & - & 1 & - & - & - \\
- & - & + & 1 & - & - & - & - & - & - \\
- & + & + & - & - & - & - & - & - & - \\
- & - & - & - & - & - & + & - & - & 2 \\
- & - & - & - & + & - & - & 1 & - & - \\
- & - & - & + & - & - & - & - & + & - \\
- & - & - & - & - & + & - & + & - & - \\
1 & - & - & - & - & - & - & - & + & - \\
- & - & - & 1 & - & - & - & - & - & + \\
- & + & + & - & - & - & - & - & - & - \\
- & - & + & - & - & - & - & - & - & 1 \\
\hline
\end{tabular}


Otras compañeras. En 1 Doronicum plantagineum + , Muscari atlanticum + , Ranunculus ficaria + . En 2 Geranium columbinum +, Orchis papilionacea + , Ornithogalum umbellatum + , Polypodium cambricum + , Stachys germanica +. En 4 Barlia robertiana +, Gladiolus italicus +, Iris planifolia +, Poa bulbosa +, Veronica cymbalaria +. En 6 Galium album +, Legousia falcata + , Silene vulgaris + , Stellaria media 1, Theligonum cynocrambe +. En 7 Colutea hispanica +, Smyrnium perfoliatum 1. En 8 Ptilostemon hispanicus +. En 9 Bupleurum spinosum + , Lepidium calycotrichum + , Leuzea conifera + , Piptatherum paradoxum +, Rhagadiolus stellatus 1, Thapsia maxima 1. En 10 Campanula rapunculus + , Cerastium boissieri + , Clinopodium vulgare arundanum + , Daphne laureola latifolia + , Euphorbia segetalis + , Orchis laxiflora + , Origanum virens + , Paeonia coriacea + .

Localidades. 1. Málaga. Alfarnatejo. Vertiente norte del Tajo del Fraile 30SUF8592. 2. Málaga. Alfarnatejo. Junto al río Sabar. 30SUF8792. 3. Málaga. Alfarnate. Frente a Venta de Alfarnate. 30SUF8794. 4. Granada. Zafarraya. Morrón del Cerrillo. 30SUF9693. 5. Málaga. Antequera. El Robledo. 30SUF7390. 6. Málaga. Colmenar. Sierra del Rey. 30SUF8391. 7. Málaga. Riogordo. Arroyo de la Cueva. 30SUF8491. 8. Granada. Alhama de Granada. Por encima de Pilas de Algaida. 30SVF0290. 9. Málaga. Villanueva del Rosario. El Hondonero. 30SUF8193 10. Málaga. Riogordo. Sierra Prieta. 30S UF7991. Cal: calizas. Mar: margas. Arc: arcillas. Mac: margocalizas.

\section{Saxifraga biternata Boiss.}

Caméfito. Endemismo antequerano (torcalense). Pedrizas. Linario-Saxifragetum biternatae. EN (LRA); VU (LFF).

\section{Saxifraga camposii Boiss. \& Reuter}

Hemicriptófito. SE de la Península Ibérica (endemismo bético). Zafarraya. Rupícola. Saxifragetum camposii. Escaso en el territorio.

\section{Teucrium chamaedrys L.}

Caméfito. Circummediterráneo. Zafarraya. Matorrales. Odontito-Thymetum baetici lavanduletosum lanatae. Muy escasa en el territorio.

\section{Thymus longiflorus Boiss. subsp. longiflorus}

Caméfito. SE de la Península Ibérica (endemismo bético). Zafarraya. Matorrales. Odontito-Thymetum baetici. Escaso en el territorio.

\section{Viola demetria Prolongo}

Terófito. $\mathrm{S}$ de la Península Ibérica y $\mathrm{N}$ de África. Zafarraya. Pastizales. Violo demetriaeIonopsidietum prolongoi. NT (LRA).

\section{Novedades y comentarios sintaxonómicos}

Se incluyen en este apartado los comentarios y descripciones de las asociaciones de interés y de las comunidades presentes en el territorio estudiado, con un número entre paréntesis que corresponde al que se les asigna en el esquema sintaxonómico. Los sintaxones de rango superior sobre los que se realizan comentarios no van numerados.

Vinco difformis-Quercetum fagineae Pérez Latorre \& Cabezudo ass. nova [Tab. 3, holotypus inv. $\left.\mathrm{n}^{\circ} 10\right]$ (5)

quercetosum fagineae

var. con Fraxinus angustifolia

Quejigares de Quercus faginea subsp. faginea que se distribuyen por el sector Antequerano (subsector Torcalense, provincia Bética) en el piso mesomediterráneo con ombrotipo húmedo-subhúmedo superior sobre sustratos básicos (calizas y margas). $\mathrm{Su}$ composición florística muestra elementos mayoritarios de Querco-Fagetea y de sus orlas espinosas caducifolias (Rhamno-Prunetea) aunque también existen especies de Quercetea ilicis. Optamos por incluirlos en Aceri-Quercion fagineae tanto por la composición florística, donde dominan las especies de QuercoFagetea, como por su estructura biestratificada, más típica de bosque caducifolio: árboles y arbustos caducifolios y sotobosque de hierbas persistentes (geófitos y hemicriptófitos).

Los bosques de Quercus faginea más próximos sintaxonómicamente a esta nueva asociación (Rivas Martínez et al., 2001; Cano et al., 2001) se diferencian claramente de los quejigares antequeranos. DaphnoAceretum granatensis Rivas Martínez 1964 es supramediterránea y presenta especies arbóreas propias (Acer granatense, Sorbus 


\begin{tabular}{|c|c|c|}
\hline Caracteres & $\begin{array}{l}\text { Viburno tini - Quercetum } \\
\text { fagineae }\end{array}$ & $\begin{array}{c}\text { Vinco difformis -Quercetum } \\
\text { fagineae }\end{array}$ \\
\hline Posición sintaxonómica & $\begin{array}{l}\text { Quercion broteroi } \\
\text { (Quercetea ilicis) }\end{array}$ & $\begin{array}{c}\text { Aceri-Quercion fagineae } \\
\text { (Querco-Fagetea) }\end{array}$ \\
\hline Distribución & Subbético & Antequerano (Torcalense) \\
\hline Ombrotipo & Subhúmedo & Húmedo-Subhúmedo superior \\
\hline Orla & $\begin{array}{l}\text { Bupleuro rigidi-Arbutetum } \\
\text { unedonis (madroñal) }\end{array}$ & $\begin{array}{c}\text { Spartio juncei-Rubetum ulmifolii } \\
\text { (zarzal) }\end{array}$ \\
\hline Matorral serial & $\begin{array}{l}\text { Thymo orospedani- } \\
\text { Cistetum clusii }\end{array}$ & $\begin{array}{l}\text { Genisto speciosae- } \\
\text { Ulicetum parviflorii }\end{array}$ \\
\hline $\begin{array}{l}\text { Especies exclusivas en cada } \\
\text { asociación (Quercetea y } \\
\text { Querco-Fagetea s. l.) }\end{array}$ & $\begin{array}{c}\text { Viburnum tinus, Bupleurum } \\
\text { fruticosum, Bupleurum rigidum, } \\
\text { Arbutus unedo, Quercus } \\
\text { coccifera, Daphne gnidium }\end{array}$ & $\begin{array}{l}\text { Ranunculus blepharicarpos, } \\
\text { Vinca difformis, Lonicera } \\
\text { etrusca, Bryonia dioica, } \\
\text { Cytisus arboreus }\end{array}$ \\
\hline
\end{tabular}

Tabla 4. Caracteres diferenciales entre los quejigales mesomediterráneos béticos de Vinco-Quercetum fagineae y Viburno-Quercetum fagineae. Main differential characters between the mesomediterranean Quercus faginea forests of the Bética phytogeographical province.

aria) y Pyro-Quercetum fagineae (Velasco 1990) Cano et al. 2001 es silicícola y LusoExtremadurense. Asensi et al. (2005) citan un Berberido hispanicae-Quercetum alpestris del Torcal de Antequera, con un solo inventario que parece corresponder a un bosque de Acer monspessulanum (comunidad comentada en este trabajo) y que sitúan en el supramediterráneo. Los quejigares más similares al ahora descrito corresponden al Viburno tini-Quercetum fagineae Cano et al. 2001 (Cano et al., 1999, Cano et al., 2001), de los que Vinco difformisQuercetum fagineae se diferencia en los caracteres indicados en la tabla 4.

Este quejigal constituye la cabecera de la criptoserie Vinco-Querceto fagineae $S$, cuya zonopotencialidad está ocupada mayoritariamente por cultivos cerealísticos. $\mathrm{Su}$ orla y primera etapa de sustitución correspondería a zarzales con "gayumbas" del Spartio-Rubetum ulmifolii, el matorral serial a Genisto-Ulicetum parviflorii y hacia suelos azonales contacta con fresnedas de Ficario-Fraxinetum angustifoliae salicetosum pedicellatae. Como vegetación exoserial más frecuente aparece una vegetación rupícola más o menos esciófila (Linario anticariaeSaxifragetum biternatae, Saxifragetum camposii) en biotopos edafoxéricos calizos y puede presentar una variante con Fraxinus angustifolia tanto en contactos con fresnedas como en suelos donde la hidromorfía es más duradera. Bioclimáticamente, puede alcanzar puntualmente el piso supramediterráneo inferior, aunque los biotopos óptimos para ello son escasos, pues están constituidos por lapiaces y otras formas kársticas con litosoles. En esas condiciones aparecen plantas como Daphne laureola o Sorbus aria que indican una posible transición a Daphno-Aceretum granatensis.

Comunidad de Acer monspessulanum y Pistacia terebinthus (6). [Tabla 5]

(Berberido hispanicae-Quercetum alpestris sensu Asensi et al., 2005: 39-40)

Comunidad de microfanerófitos caducifolios (A. monspessulanum, Pistacia terebinthus), muy restringidos a biotopos kársticos: base de cantiles y borde de canchales 
Tabla 5

Comunidad de Acer monspessulanum y Pistacia terebinthus (6)

(Querco-Fagetea, Quercetalia pubescentispetraeae, Aceri-Quercion fagineae)

\begin{tabular}{lcr} 
INVENTARIO N $^{\text {o }}$ & 1 & 2 \\
Orientación & $\mathrm{NW}$ & $\mathrm{N}$ \\
Inclinación $\left.^{\circ}{ }^{\circ}\right)$ & 40 & 5 \\
Área $\left(\mathrm{m}^{2}\right)$ & 300 & 100 \\
Litología & Cal/Mar & $\mathrm{Cal}$ \\
Altitud $(\mathrm{m})$ & 940 & 1000 \\
Altura vegetación (m) & 8 & 6 \\
Características de comunidad & & \\
Acer monspessulanum & 3 & 3 \\
Paeonia broteroi & + & + \\
Pistacia terebinthus & 2 & - \\
Características de unidades superiores & \\
(Querco-Fagetea y Rhamno-Prunetea) & \\
Hedera helix & + & 2 \\
Quercus faginea & + & + \\
Tamus communis & + & 1 \\
Crataegus monogyna & - & + \\
Helleborus foetidus & + & - \\
Lonicera etrusca & - & + \\
Rosa pouzinii & + \\
Rubus ulmifolius & + & - \\
Vinca difformis & - & 1 \\
Compañeras & 1 & - \\
Asparagus acutifolius & & \\
Rhamnus alaternus & + & 1 \\
\hline & + & + \\
\hline
\end{tabular}

Otras compañeras. En 1 Arabis verna + , Bellis perennis + , Cerastium boissieri 1, Ptilostemon hispanicus + , Ruscus aculeatus 1, Hyacinthoides hispanica +. En 2 Briófitos 2, Geranium lucidum 2, Geranium purpureum +, Rhagadiolus edulis 1, Scandix pecten-veneris 1, Scilla peruviana + . Localidades. 1. Málaga. Alfarnatejo. Entre Tajo de Gomer y El Fraile. 30SUF8691. 2. Málaga. Villanueva del Rosario. El Hondonero. 30SUF 8193. Cal: calizas. Mar: margas.

y lapiaces, dejando los suelos profundos a VincoQuercetum fagineae. Se desarrolla en los pisos meso y supramediterráneo torcalenses bajo ombrotipo húmedo-subhúmedo. Posiblemente se trata de una asociación relicta y fragmentaria que podría sustituir en el piso supramediterráneo de este subsector al Daphno-Aceretum granatensis, ya que no se ha detectado Acer granatense. Se distingue de Vinco-Quercetum fagineae por la gran preponderancia de las especies de Querco-Fagetea y RhamnoPrunetea sobre las de Quercetea, y la existencia residual de Quercus faginea. El sintaxon propuesto por Asensi et al. (2005) para el Torcal de Antequera (subsector Torcalense) con un solo inventario, parece corresponder a un aceral de $A$. monspessulanum con composición florística prácticamente idéntica a la comunidad aquí descrita (Crataegus granatensis, aparece en todo el subsector en quejigales y zarzales junto a C. monogyna).

Genisto speciosae-Cytisetum reverchonii $\mathrm{F}$. Valle 1987 (15)

Los escobonales basófilos béticoorientales supra-mesomediterráneos alcanzan en el subsector Torcalense su límite más occidental, aunque de modo muy fragmentario y empobrecido.

Localidad: Granada. Zafarraya. Frente al Cerro del Moro. 30SUF9494. Orientación N, inclinación $30^{\circ}$, área $50 \mathrm{~m}^{2}$, calizas y dolomías, $1150 \mathrm{~m}$, altura vegetación $3 \mathrm{~m}$. Características: Cytisus scoparius subsp. reverchonii 4, Genista cinerea +. Compañeras: Crataegus granatensis 3, Ulex parviflorus 2, Berberis hispanica 1, Ranunculus blepharicarpos 1, Rubus ulmifolius 1, Andryala ramosissima + , Armeria villosa subsp. longiaristata + , Cerastium gibraltaricum var. lanuginosum + , Clematis flammula +, Festuca scariosa +, Prunus dulcis + , Ptilostemon hispanicus + , Quercus rotundifolia + , Rhamnus saxatilis + , Rosa canina + , Thymus mastichina + .

Odontito purpureae-Thymetum baetici Esteve \& López Guadalupe 1977 (16) thymetosum baetici

lavanduletosum lanatae Pérez Latorre \& Cabezudo in Pérez Latorre, D. Navas, O. Gavira, G. Caballero \& Cabezudo 2004 [Tab. 6] 
Tabla 6

Odontito purpureae-Thymetum baetici Esteve \& López Guadalupe 1977 (16)

thymetosum baetici, lavanduletosum lanatae Pérez Latorre \& Cabezudo in Pérez Latorre, D. Navas, O. Gavira, G. Caballero \& Cabezudo 2004

(Cisto-Micromerietea, Rosmarinetalia, Saturejo micranthae-Thymbrion capitati)

\begin{tabular}{|c|c|c|c|c|c|}
\hline INVENTARIO N ${ }^{\circ}$ & 1 & 2 & 3 & 4 & 5 \\
\hline Orientación & NW & $\mathrm{N}$ & SE & SW & $\mathrm{S}$ \\
\hline Inclinación $\left({ }^{\circ}\right)$ & 25 & 35 & 10 & 45 & 45 \\
\hline Área $\left(\mathrm{m}^{2}\right)$ & 200 & 150 & 200 & 100 & 100 \\
\hline Litología & Dol & Dol & Dol & Dol & Cal \\
\hline Altitud (m) & 1160 & 930 & 700 & 640 & 700 \\
\hline Altura vegetación $(\mathrm{cm})$ & 70 & 70 & 60 & 100 & 60 \\
\hline \multicolumn{6}{|c|}{ Características y diferenciales de asociación } \\
\hline Thymus longiflorus & 1 & + & 2 & 2 & 1 \\
\hline Thymus baeticus & - & 1 & 1 & 2 & 2 \\
\hline Viola arborescens & - & - & - & + & - \\
\hline \multicolumn{6}{|c|}{ Diferenciales de subasociación } \\
\hline Lavandula lanata & 2 & 3 & - & - & - \\
\hline Ptilostemon hispanicus & 1 & 1 & + & 1 & - \\
\hline \multicolumn{6}{|c|}{ Características de unidades superiores } \\
\hline Teucrium lusitanicum & 1 & 1 & 2 & - & + \\
\hline Cistus albidus & 2 & - & + & + & - \\
\hline Fumana thymifolia & - & - & 1 & 1 & 1 \\
\hline Rosmarinus officinalis & + & - & - & 3 & 4 \\
\hline Helianthemum appeninum & 1 & + & - & - & - \\
\hline Helianthemum hirtum & + & + & - & - & - \\
\hline Scabiosa turolensis & - & 1 & 1 & - & - \\
\hline
\end{tabular}

Otras características. En 1 Anthyllis vulneraria +, Argyrolobium zanonii +, Linum suffruticosum 1, Staehelina dubia 1, Teucrium chamaedrys +. En 2 Euphorbia nicaeensis + . En 3 Convolvulus lanuginosus +, Cytisus fontanesii + , Helianthemum marifolium + , Thesium humile 1. En 4 Cistus clusii 3, Coris monspeliensis 1, Thymelaea argentata + . En 5 Satureja graeca + , Thymelaea hirsuta.+ Compañeras

Ulex parviflorus

Brachypodium retusum

Phlomis lychnitis

Santolina chamaecyparissus

Helichrysum stoechas

Phlomis purpurea

Asparagus horridus

Chamaerops humilis

Daphne gnidium

Genista umbellata equisetiformis

Rhamnus velutinus

Dianthus broteri

Stipa tenacissima

\begin{tabular}{lllll}
3 & 4 & 3 & + & 1 \\
3 & 1 & 2 & 2 & 2 \\
1 & - & 1 & + & 1 \\
+ & 1 & - & + & + \\
- & 1 & + & + & - \\
+ & - & 1 & + & 2 \\
- & - & 1 & + & + \\
- & - & 1 & + & 1 \\
- & 1 & + & 1 & - \\
+ & - & 1 & - & + \\
- & - & + & + & + \\
- & - & 1 & - & 1 \\
- & - & - & 2 & 1 \\
\hline
\end{tabular}

Otras compañeras. En 1 Bellis sylvestris + , Carlina corymbosa + , Cephalaria leucantha 1 , Leuzea conifera + , Orchis italica +, Quercus rotundifolia +, Thapsia villosa + . En 2 Andryala ramosissima 1, Asphodelus albus +, Cerastium boissieri +, Chaenorrhinum macropodum macropodum +, Crataegus monogyna +, Hippocrepis rupestris + , Ophrys lutea + , Ranunculus rupestris + , Salvia candelabrum + , Sanguisorba minor +. En 3 Allium sphaerocephalon +, Asparagus albus + , Asperula aristata scabra +, Cuscuta epithymum +, Eryngium campestre + , Phagnalon saxatile + , Reseda phyteuma + . En 4 Juniperus oxycedrus + , Klasea flavescens + , Olea europaea sylvestris + . En 5 Carthamus arborescens + , Hyparrhenia hirta + , Lavandula multifida + , Malva hispanica + , Polygala rupestris + , Retama sphaerocarpa + .

Localidades. 1. Málaga. Alfarnate. Sierra del Gallo. 30SUF8793. 2. Granada. Alhama de Granada. Boquete de Zafarraya. 30SUF9990. 3. Málaga. Alcaucín. Subida al Boquete. 30SUF9989. 4. Málaga. Alcaucín. Loma de Las Monjas. 30SVF0087. 5. Málaga. Alcaucín. Mesa de Zalia. 30SUF9887. Dol: dolomías. Cal: calizas. 
Matorrales bien desarrollados en los subsectores Alpujarreño y Almijarense de la provincia Bética. Son de interés fitogeográfico en la zona, ya que constituyen una continuación de su área de distribución típica, con límite occidental en las sierras Torcalenses estudiadas (unidad de Zafarraya). Se desarrolla como la subasociación típica en el piso termomediterráneo sobre calizas y dolomías y como subasociación lavanduletosum lanatae sobre dolomías en el piso mesomediterráneo, siempre en laderas térmicas, abiertas hacia la influencia del mar Mediterráneo, ya que es sustituido en el mesomediterráneo, más continentalizado, por el Thymo gracilisLavanduletum lanatae.

\section{Genisto speciosae-Ulicetum parviflorii Rivas}

Goday \& Rivas-Martínez ex Pérez Latorre y Cabezudo nom. inv. propos. (18) [Tab. 7]

(Ulici parviflorii-Genistetum speciosae Rivas Goday \& Rivas-Martínez 1968)

[Lectotypus inv. 3 tab. 20 in Rivas Goday \& Rivas Martínez in Anales Inst. Bot. Cavanilles 25:5-180 (1968) designado aquí]

Asociación originalmente descrita con inventarios realizados en tres zonas muy distintas biogeográficamente (Rivas Goday \& Rivas Martínez, 1968). Los inventarios 1 a 3 son del subsector Antequerano, los del 4 a 7 del sector Subbético y los del 8 al 11 del subsector Torcalense (sobre dolomías). Los propios autores (Rivas Goday \& Rivas Martínez, op. cit.: 134) afirman que "es comunidad susceptible de desmembrase e integrar nueva unidad". Tras realizar inventarios en dos de las zonas estudiadas por Rivas Goday y Rivas Martínez, llegamos a la conclusión de que a cada zona corresponde una asociación distinta. Los inventarios 8 a 10 de Los Alazores (MálagaGranada) sobre dolomías y con Lavandula lanata como bioindicador, pertenecen al Thymo gracilis -Lavanduletum lanatae. El inventario 11 del Boquete de Zafarraya (Granada) sobre dolomías corresponde a un OdontitoThymetum baetici lavanduletosum lanatae (ver. tab. 5). Los inventarios antequeranos 1 y 2 sobre margocalizas corresponden al GenistoCytisetum fontanesii en base a su composición florística. El inventario 3, de las Pedrizas, sin Genista umbellata ni Cytisus fontanesii, es el que representa mejor a Genisto-Ulicetum parviflorii, ya que los inventarios de Jaén parecen corresponder a Retamo-Genistetum speciosae (sobre todo el 5). El inventario de Rute (Córdoba) se asemeja más a un TeucrioCoridothymetum capitati (Rivas Goday \& Rivas-Martínez 1968) Asensi \& Díez-Garretas 1989 a la vista de la tabla de Gómez Mercado et al. (2000) en la Subbética cordobesa.

La asociación se fundamenta por tanto en un solo inventario (el número 3 de la tabla original). Se trata de un matorral que debe ser incluido en el orden Rosmarinetalia, alianza Saturejo-Thymbrion como los propios autores hicieron. Al no designar los autores el tipo y que solo queda el inv. 3 como representante de ésta asociación, elegimos dicho inventario como lectótipo de la misma. Se propone la inversión del nombre, ya que Ulex parviflorus es siempre dominante sobre Genista speciosa. Esta asociación es un matorral con distribución en el subsector Torcalense, sobre calizas y margocalizas jurásicas, y diferenciable de Genisto-Cytisetum fontanesii que es propio del subsector Antequerano y se desarrolla sobre margas y margocalizas triásicas (a veces abigarradas con yesos). Con las demás asociaciones citadas anteriormente, la ausencia de Thymus zygis subsp. gracilis y Lavandula lanata lo diferencian del Thymo gracilisLavanduletum lanatae y la ausencia de Thymus longiflorus, Lavandula lanata y Santolina chamaecyparissus del Odontito-Thymetum baetici.

Comunidad de Ptilotrichum spinosum BC (19)

La existencia en el subsector Torcalense 
Tabla 7

Genisto speciosae-Ulicetum parviflorii Rivas Goday \& Rivas Martínez ex Pérez Latorre \& Cabezudo nom. inv. propos. (18)

(Cisto-Micromerietea, Rosmarinetalia, Saturejo micranthae-Thymbrion capitati)

\begin{tabular}{|c|c|c|c|c|}
\hline INVENTARIO N ${ }^{\circ}$ & 1 & 2 & 3 & 4 \\
\hline Orientación & SW & $\mathrm{S}$ & NW & $\mathrm{S}$ \\
\hline Inclinación $\left({ }^{\circ}\right)$ & 5 & 10 & 10 & - \\
\hline Área $\left(\mathrm{m}^{2}\right)$ & 150 & 250 & 250 & 100 \\
\hline Litología & $\mathrm{Cal}$ & $\mathrm{Mar} / \mathrm{Cal}$ & Cal & - \\
\hline Altitud (m) & 750 & 1140 & 1300 & 850 \\
\hline Altura vegetación (cm) & 80 & 70 & 80 & - \\
\hline \multicolumn{5}{|c|}{ Características y diferenciales de asociación } \\
\hline Ulex parviflorus & 4 & 5 & 3 & 1 \\
\hline Genista cinerea subsp. speciosa & + & + & - & + \\
\hline Santolina canescens & - & 1 & - & 2 \\
\hline \multicolumn{5}{|l|}{ Características de unidades superiores } \\
\hline Cistus albidus & 2 & 1 & 1 & 1 \\
\hline Argyrolobium zanonii & - & + & + & + \\
\hline Rosmarinus officinalis & 2 & 1 & - & 2 \\
\hline Thymbra capitata & 1 & 1 & - & - \\
\hline Fumana thymifolia & - & + & - & + \\
\hline Helianthemum hirtum & - & 1 & + & + \\
\hline Anthyllis vulneraria subsp. arundana & - & - & + & - \\
\hline Asperula aristata subsp. scabra & - & - & 1 & - \\
\hline Cistus clusii & - & - & - & + \\
\hline Heliantheтит appeninum & - & - & 1 & - \\
\hline Helianthemum racemosum & - & - & - & + \\
\hline Lithodora fruticosa & - & - & + & - \\
\hline Satureja graeca & + & - & - & - \\
\hline Staehelina dubia & - & - & - & 1 \\
\hline Teucrium capitatum & 1 & - & - & - \\
\hline Teucrium similatum & - & - & + & - \\
\hline Thymus baeticus & - & - & - & 2 \\
\hline \multicolumn{5}{|l|}{ Compañeras } \\
\hline Thymus mastichina & 1 & 1 & + & + \\
\hline Phlomis lychnitis & - & + & 1 & + \\
\hline Phlomis purpurea & 2 & 1 & - & 2 \\
\hline Ptilostemon hispanicus & - & 1 & 1 & 1 \\
\hline Asparagus acutifolius & + & - & - & + \\
\hline Brachypodium retusum & - & 1 & - & 2 \\
\hline Crataegus monogyna & - & + & 1 & - \\
\hline Daphne gnidium & - & + & - & 1 \\
\hline Festuca scariosa & - & + & 1 & - \\
\hline Helichrysum stoechas & + & - & 1 & - \\
\hline Quercus rotundifolia & - & + & + & - \\
\hline Ruta angustifolia & - & + & - & + \\
\hline
\end{tabular}

Otras compañeras. En 1 Convolvulus althaeoides + , Genista umbellata equisetiformis 2, Olea europaea sylvestris + , Osyris alba 1, Polygala rupestris +, Retama sphaerocarpa 1 , Rhamnus alaternus 1 , Rhamnus oleoides + , Rosa canina +, Spartium junceum + . En 2 Carex hallerana +, Carlina corymbosa 1, Crocus nudiflorus + , Dactylis hispanica + . En 3 Anacamptis pyramidalis + , Anarrhinum bellidifolium + , Dictamnus albus + , Euphorbia nicaeensis 1, Festuca capillifolia + , Helleborus foetidus + , Paeonia broteroi + , Pinus sylvestris +, Pinus nigra 1, Quercus faginea +, Rhamnus saxatilis +. En 4 Asparagus albus + , Asteriscus aquaticus +, Avena bromoides + , Brachypodium dystachion + , Bupleurum acutifolium 1, Cistus monspeliensis 1, Cleonia lusitanica +, Crupina vulgaris 1, Erucastrum virgatum subsp. baeticum + , Festuca triflora 1, Leuzea conifera + , Linum strictum + , Malva althaeoides + , Onobrychis argentea + , Plantago albicans + , Plantago lagopus + , Psoralea bituminosa 1, Scabiosa sicula + , Scilla maritima + .

Localidades. 1. Málaga. Antequera. El Robledo. 30SUF7290. 2. Málaga. Alfarnate. Sierra Gorda. 3. Málaga. Alfarnate. Subida al Vilo. 30SUF9092 4. Málaga. Cerca del Puerto de Las Pedrizas (Rivas Goday \& Rivas Martínez, 1968, Tab. 29 inv. 3). Cal: calizas. Mar: margas. 
del piso supramediterráneo se corrobora no tanto por la altitud, ya que las sierras solo alcanzan $1500 \mathrm{~m}$ en el territorio estudiado, sino por la orientación de las crestas norte-sur, muy batidas por el viento, y por la litología de lapiaces y roquedos que favorecen a las especies pulvinulares de Xeroacantho-Erinaceion.

Localidad: Granada. Zafarraya. La Torca. 30SUF9393. Orientación -, inclinación -, área $125 \mathrm{~m}^{2}$, calizas, $1380 \mathrm{~m}$, altura vegetación $25 \mathrm{~cm}$. Característica: Ptilotrichum spinosum 2. Unidades superiores: Cerastium boissieri + , Scabiosa turolensis subsp. gross ${ }^{+}$. Compañeras: Cynoglossum arundanum +, Dactylis glomerata subsp. hispanica + , Dianthus pungens + , Erodium cheilanthifolium + , Euphorbia segetalis + , Festuca scariosa 1, Helleborus foetidus 1, Koeleria vallesiana + , Rhamnus saxatilis + , Sedum acre + , Thymus mastichina + .

\section{Comunidad de Erodium primulaceum BC}

Los majadales constituyen una unidad de vegetación muy extendida por el territorio y de gran importancia paisajística por su uso antrópico. Los incluimos provisionalmente en esta comunidad, aunque un estudio más detallado en todo el subsector Torcalense quizá podría demostrar algún rango sintaxonómico.

Localidad: Málaga. Alfarnate. Sierra de Vilo. 30SUF8993. Orientación NW, inclinación $5^{\circ}$, área $10 \mathrm{~m}^{2}$, calizas, altitud $1200 \mathrm{~m}$, altura vegetación $10 \mathrm{~cm}$. Características de alianza y unidades superiores: Poa bulbosa 3, Erodium primulaceum 1, Paronychia argentea + , Trifolium tomentosum 1. Compañeras: Plantago lagopus 3, Rumex bucephalophorus 2, Trifolium stellatum 1, Anthemis arvensis 2, Leontodon longirrostris 2, Bromus rubens 1, Carduus tenuiflorus 1, Crambe filiformis + , Dactylis hispanica + , Desmazeria rigida + , Lagurus ovatus + , Linaria oblongifolia + , Medicago minima + , Papaver rhoeas + , Petrorrhagia nanteuillii + , Polygala rupestris + , Trifolium campestre 1, Trifolium scabrum +

Campanulion velutinae Martínez Parras \& Peinado 1990

En el territorio existen tres comunidades de esta alianza localizadas en el mismo piso bioclimático pero en distintos biotopos en función de la nitrificación y la heliofilia. LinarioCentaureetum clementei es subnitrófila y heliófila, mientras que Biscutello-Saxifragetum reuteranae es menos heliófila e incluso esciófila y vive en grietas escasamente nitrificadas de acantilados de gran superficie. LinarioSaxifragetum biternatae es completamente esciófila y de grietas no nitrificadas en pequeños acantilados, incluso roquedos aislados.

A continuación se comentan los aspectos más relevantes de las tres asociaciones:

\section{Biscutello frutescentis-Saxifragetum reuteranae}

Socorro \& Marín Calderón 1983 saxifragetosum reuteranae (39) [Tab. 8]

Comunidad rupícola mesomediterránea basófila, rica en especies de Campanulion velutinae. En nuestro territorio presenta una variante termófila con Hippocrepis rupestris $\mathrm{y}$ otra a mayor altitud que se enriquece en Erodium cheilanthifolium. Creemos que la variante subrupícola de esta asociación dada por los autores se basa en especies que pueden aparecer también en fisuras con verticalidad (rupícolas).

\section{Linario anticariae-Saxifragetum biternatae}

Esteve \& López Guadalupe 1973 (40) [Tab. 9]

Asociación saxícola mesomediterránea y basófila clásica del Torcal de Antequera (zona central de la Cordillera Antequerana) que alcanza el territorio de estudio en su, hasta ahora, localización más oriental.

\section{Linario anticariae-Centaureetum clementei}

Cabezudo \& Pérez Latorre ass. nova (41)

[Tab. 10, holotypus inv. $n^{\circ}$ 5] 
Tabla 8

Biscutello frutescentis-Saxifragetum reuteranae Socorro \& Marín Calderón 1983 (39) saxifragetosum reuteranae, var. de Erodium cheilanthifolium, var. de Hippocrepis rupestris (Asplenietea, Asplenietalia, Campanulion velutinae, Campanulenion velutinae)

\begin{tabular}{|c|c|c|c|c|c|}
\hline INVENTARIO N & 1 & 2 & 3 & 4 & 5 \\
\hline Orientación & $\mathrm{S}$ & $\mathrm{S}$ & W & $\mathrm{N}$ & $\mathrm{N}$ \\
\hline Inclinación $\left(^{\circ}\right)$ & 80 & 90 & 90 & 80 & 90 \\
\hline Área $\left(m^{2}\right)$ & 4 & 8 & 10 & 4 & 8 \\
\hline Litología & Cal & Cal & Cal & Cal & Cal \\
\hline Altitud (m) & 920 & 960 & 920 & 1000 & 1000 \\
\hline Altura vegetación (cm) & 10 & 15 & 25 & 15 & 20 \\
\hline \multicolumn{6}{|c|}{ Características y diferenciales de asociación } \\
\hline Biscutella frutescens & - & - & 1 & + & 1 \\
\hline Centaurea prolongoi & - & + & - & - & + \\
\hline Hesperis laciniata & - & - & + & - & + \\
\hline \multicolumn{6}{|l|}{ Diferenciales de variantes } \\
\hline Erodium cheilanthifolium & - & - & - & 1 & + \\
\hline Hippocrepis rupestris & 1 & + & - & - & - \\
\hline \multicolumn{6}{|c|}{ Características de unidades superiores } \\
\hline Ceterach officinarum & + & + & + & + & + \\
\hline Crepis albida & - & + & 1 & 2 & 1 \\
\hline Campanula velutina & 1 & + & + & - & - \\
\hline Chaenorrhinum villosum & 2 & - & - & 2 & 1 \\
\hline Linaria anticaria & - & - & + & + & + \\
\hline Melica minuta & 1 & 1 & - & 1 & - \\
\hline Silene andryalifolia & - & - & 1 & + & 1 \\
\hline Polygala rupestris & + & + & - & - & - \\
\hline Cheilanthes acrosticha & + & - & - & - & - \\
\hline \multicolumn{6}{|l|}{ Compañeras } \\
\hline Galium lucidum & - & - & 1 & + & + \\
\hline Sedum dasyphyllum & + & + & - & - & + \\
\hline Antirrhinum hispanicum & - & 1 & - & - & + \\
\hline Bupleurum gibraltaricum & - & + & + & - & - \\
\hline Cerastium boissieri & - & - & - & + & + \\
\hline Fumaria macrosepala & + & + & - & - & - \\
\hline Lobularia maritima & + & + & - & - & - \\
\hline Ranunculus blepharicarpos & - & - & - & 1 & 1 \\
\hline Rhamnus myrtifolius & + & + & - & - & - \\
\hline
\end{tabular}

Otras compañeras. En 1 Dipcadi serotinum 1, Erodium cicutarium +, Parietaria mauritanica + , Phagnalon saxatile +, Sedum sediforme +. En 2 Asperula aristata subsp. scabra 1, Calendula suffruticosa +, Ficus carica + , Hedypnois cretica + , Lapiedra martinezii + , Putoria calabrica 1 . En 3 Dianthus broteri + , Ephedra fragilis + , Sedum mucizonia + , Umbilicus rupestris + . En 4 Coronilla glauca + , Dianthus pungens subsp. brachyanthus + , Geranium purpureum + , Lactuca tenerrima + , Prunus mahaleb + . En 5 Armeria villosa subsp. longiaristata + , Erinacea anthyllis + , Polypodium cambricum + .

Localidades. 1. Málaga. Alcaucín. Boquete de Zafarraya, antigua vía del tren. 30SUF9989. 2. Málaga. Alfarnatejo. Tajo de El Fraile. 30SUF8791. 3. Granada. Alhama de Granada. Boquete de Zafarraya. 30SVF0090. 4. Málaga. Alfarnatejo. Tajo de Doña Ana. 30SUF8691. 5. Málaga. Riogordo. Tajos de Gomer. 30SUF8691. Cal. calizas. 
Centaureenion clementei Cabezudo \& Pérez Latorre suball. nova

Las comunidades rupícolas heliófilas y subnitrófilas de grandes hemicriptófitos caracterizadas por Centaurea clementei alcanzan el sector Antequerano en la unidad Pedrizas del subsector Torcalense (Sierra del Co). En la descripción original (Asensi, 1976) de la asociación Sarcocapno baeticiCentaureetum clementei de Sierra de las Nieves (Sector Rondeño, Málaga) aparecen mezcladas especies con distintos tipos biológicos y propias de distintos biotopos, mezcla producida posiblemente por la dificultad que entraña en medios rupestres diferenciar las distintas ecologías. Sarcocapnos baetica es un nanocaméfito escionitrófilo y espeluncícola (de extraplomos) de pequeñas grietas (Petrocoptido-Sarcocapnetea), mientras que Centaurea clementei es un caméfito rosulado de gran tamaño, heliófilo y de grandes grietas (Asplenietea), a veces no verticales (Pérez Latorre et al., 1998: 158), ecología y tipos biológicos que se repiten en el territorio de estudio y también en el sector Subbético (Gómez Mercado et al., 2000). No nos parece adecuado por tanto incluir SarcocapnoCentaureetum clementei en Sarcocapnetalia enneaphyllae como proponen Rivas Martínez et al. $(2001,2002)$ sino en Campanulion velutinae (Asplenietea).

Los inventarios procedentes del subsector Torcalense y del sector Subbético ponen de manifiesto una composición florística distinta a la de los inventarios Rondeños, por lo que proponemos una nueva asociación: Linario anticariae-Centaureetum clementei ass. nova. Las especies diferenciales frente a la asociación de Sierra de las Nieves (Tolox) son Linaria anticaria, Biscutella frutescens y Antirrhinum graniticum subsp. boissieri, estando ausentes en la nueva asociación Asplenium trichomanes, Putoria calabrica, Umbilicus rupestris y, sobre todo, Sarcocapnos baetica (exclusiva de los inventarios del Tajo de la Caína, Tolox) y que no aparece en los inventarios de Grazalema ni de otras zonas de la Sierra de Tolox (Socorro \& Marín Calderón, 1983; Pérez Latorre et al., 1998). La nueva asociación, al contrario que Sarcocapno-Centaureetum, es en general más termófila (entre 700 y $1200 \mathrm{~m}$, piso mesomediterráneo subhúmedo) y presenta frecuentemente especies de fanerófitos rupícolas como Rhamnus myrtifolius y Bupleurum gibraltaricum. Finalmente, agrupamos estas dos asociaciones con Centaurea clementei, en una nueva subalianza Centaureenion clementei suball. nova, dentro de Campanulion velutinae, de areal bético más occidental (Rondeño, Subbético cordobés y Torcalense) que no alcanza las serranías béticas orientales y ocupa posiciones más heliófilas y xerófilas que el resto de asociaciones (Campanulenion velutinae) del territorio aquí tratado.

Saxifragetum camposii Cuatrecasas ex Martínez Parras y Peinado Lorca 1990 (42)

En el subsector Torcalense se encuentran las únicas localidades conocidas hasta la fecha, en la provincia de Málaga, de esta asociación supramediterránea, basófila, bética.

Localidad: Málaga. Alfarnate. La Torca. 30SUF9294. Orientación NE, Inclinación $90^{\circ}$, área $6 \mathrm{~m}^{2}$, calizas, altitud $1340 \mathrm{~m}$, altura vegetación $10 \mathrm{~cm}$. Características: Saxifraga camposii 1. Unidades superiores: Biscutella frutescens 2, Crepis albida 1, Linaria anticaria + , Silene andryalifolia 2. Compañeras: Antirrhinum graniticum subsp. boissieri + , Armeria villosa subsp. longiaristata + , Bryophyta 4, Cerastium boissieri +, Fumaria macrosepala +, Geranium purpureum 1, Sedum album + , Stachys circinata + , Umbilicus rupestris + .

\section{Elymo repentis-Phalaridetum coerulescentis} Pérez Latorre, Galán \& Cabezudo in Pérez Latorre, P. Navas, D. Navas, Y. Gil y Cabezudo 1998 (47) 
Tabla 9

\begin{tabular}{|c|c|c|}
\hline \multicolumn{3}{|c|}{$\begin{array}{l}\text { Linario anticariae-Saxifragetum biternatae } \\
\text { Esteve \& López Guadalupe } 1973 \text { (40) } \\
\text { (Asplenietea, Asplenietalia, Campanulion } \\
\text { velutinae, Campanulenion velutinae) }\end{array}$} \\
\hline INVENTARIO N ${ }^{\circ}$ & 1 & 2 \\
\hline Orientación & $\mathrm{N}$ & $\mathrm{N}$ \\
\hline Inclinación $\left({ }^{\circ}\right)$ & 90 & 90 \\
\hline Área $\left(m^{2}\right)$ & 2 & 4 \\
\hline Litología & $\mathrm{Cal}$ & Cal \\
\hline Altitud (m) & 820 & 940 \\
\hline Altura vegetación $(\mathrm{cm})$ & 15 & 30 \\
\hline \multicolumn{3}{|c|}{ Características y diferenciales de asociación } \\
\hline Saxifraga biternata & + & 1 \\
\hline Linaria anticaria & + & + \\
\hline \multicolumn{3}{|c|}{ Características de unidades superiores } \\
\hline Melica minuta & 1 & + \\
\hline Ceterach officinarum & + & - \\
\hline Biscutella frutescens & - & + \\
\hline Silene andryalifolia & - & + \\
\hline \multicolumn{3}{|l|}{ Compañeras } \\
\hline Stachys circinata & + & 1 \\
\hline Galium verrucosum & + & + \\
\hline Sedum mucizonia & + & - \\
\hline Centranthus calcitrapae & + & - \\
\hline Umbilicus rupestris & - & + \\
\hline
\end{tabular}

Localidades. 1. Málaga. Antequera. El Carrascal. 30SUF6792. 2. Málaga. Colmenar. Sierra del Rey. 30SUF8391. Cal: calizas.

Asociación de interés en el territorio estudiado pues se trata de la primera localidad fuera del sector Rondeño de donde fue descrita. En el subsector Torcalense también se presenta como un prado hidrófilo de desarrollo primaveral sobre suelos vérticos en la serie de las fresnedas de Ficario-Fraxineto angustifoliae salicetoso pedicellatae $S$ y de los quejigares sobre margas hidromorfas del Vinco-Querceto fagineae $S$ faciación con Fraxinus angustifolia. También es probable su existencia en el contiguo sector Subbético (subbéticas cordobesas, Sierra de Cabra) a la vista de los inventarios de Gómez Mercado et al. (2000) (comunidad de Gaudinia fragilis y Agrostis castellana).
Localidad: Málaga. Riogordo. Hospedería E1 Retamar. 30SUF8390. Orientación S, Inclinación $5^{\circ}$, Área $100 \mathrm{~m}^{2}$, margas, altitud 760 $\mathrm{m}$, altura vegetación $100 \mathrm{~cm}$. Características: Achillea ageratum 2, Elymus repens + , Phalaris coerulescens 2. Unidades superiores: Crepis vesicaria 3, Hordeum bulbosum 3, Scirpus holoschoenus 2, Festuca arundinacea subsp. atlantigena 1, Ranunculus macrophyllus 1, Mentha rotundifolia + , Scilla peruviana + . Compañeras: Anacamptis pyramidalis +, Carduncellus caeruleus + , Centaurea pullata subsp. baetica + , Dactylis glomerata + , Daucus carota subsp. maximus + , Fraxinus angustifolia ,+ Plantago lagopus + .

\section{Callitricho stagnalis-Ranunculetum saniculifolii Galán in A. V. Pérez, Galán, P. Navas, D. Navas, Y. Gil \& Cabezudo 1999 (48)}

Asociación de gran interés en el territorio pues marca los últimos vestigios de la antiguamente amplia vegetación hidrofítica que sin duda existió en el gran Poljé de Zafarraya, hoy día cultivado en un $99 \%$ de su superficie.

Localidad: Granada. Zafarraya. Llanos de Zafarraya, Lagunajo del Concejo. 30SUF9993. Orientación -, Inclinación $\left({ }^{\circ}\right)$-, área $4 \mathrm{~m}^{2}$, arcillas, altitud $920 \mathrm{~m}$, altura vegetación 15 $\mathrm{cm}$. Características: Ranunculus peltatus subsp. saniculifolius 3. Compañeras: Glyceria notata 1, Rumex crispus 1, Ranunculus repens + .

\section{Series de vegetación}

Para entender la distribución de la vegetación en el subsector Torcalense hay que partir de la base de la gran fragmentación teselar del territorio, que causa un cambio de zonopotencialidad en muy pequeñas áreas. De este modo, son muy escasas las grandes extensiones que corresponden a una sola serie de vegetación y son más frecuentes mosaicos teselares correspondientes a varias series o faciaciones, mezcladas con vegetación exoserial. 
Tabla 10

Linario anticariae-Centaureetum clementei Pérez Latorre \& Cabezudo ass. nova (Asplenietea, Asplenietalia, Campanulion velutinae, Centaureenion clementei)

\begin{tabular}{|c|c|c|c|c|c|c|}
\hline INVENTARIO N ${ }^{\circ}$ & 1 & 2 & 3 & 4 & 5 & 6 \\
\hline Orientación & $\mathrm{N}$ & $\mathrm{E}$ & NW & NW & $\mathrm{N}$ & $\mathrm{S}$ a SE \\
\hline Inclinación $\left(^{\circ}\right)$ & 90 & 90 & 85 & 90 & 90 & 90 \\
\hline Área $\left(\mathrm{m}^{2}\right)$ & 10 & 25 & 4 & 16 & 50 & 5 a 10 \\
\hline Litología & Cal & Dol & Cal & Cal & $\mathrm{Cal}$ & Cal \\
\hline Altitud (m) & 920 & 1200 & 820 & 880 & 700 & 1350 \\
\hline Altura vegetación (cm) & 40 & 50 & - & - & 40 & - \\
\hline \multicolumn{7}{|l|}{ Características de asociación y subalianza } \\
\hline Centaurea clementei & 1 & 2 & 2 & 1 & 2 & IV \\
\hline Biscutella frutescens & + & - & - & 1 & 1 & - \\
\hline Linaria anticaria & + & - & 1 & - & 1 & - \\
\hline Antirrhinum graniticum subsp. boissieri & - & 1 & - & + & + & - \\
\hline \multicolumn{7}{|c|}{ Característica y diferenciales de Sarcocapno-Centaureetum clementei } \\
\hline Sarcocapnos baetica subsp. baetica & - & - & - & - & - & V \\
\hline Asplenium trichomanes & - & - & - & - & - & V \\
\hline Putoria calabrica & - & - & - & - & - & II \\
\hline Umbilicus rupestris & - & - & - & - & - & IV \\
\hline \multicolumn{7}{|l|}{ Unidades superiores } \\
\hline Silene andryalifolia & + & + & + & - & + & IV \\
\hline Campanula velutina & - & - & 1 & 1 & + & $\mathrm{V}$ \\
\hline Chaenorrhinum villosum & + & - & + & - & + & V \\
\hline Asplenium ceterach & - & + & + & + & - & - \\
\hline Melica minuta & + & - & - & - & - & IV \\
\hline Sanguisorba minor var. rupicola & - & + & + & - & - & - \\
\hline Asplenium petrarchae & - & + & - & - & - & - \\
\hline Crepis albida & + & - & - & - & - & - \\
\hline Jasonia glutinosa & - & - & - & + & - & - \\
\hline Teucrium rotundifolium & - & - & - & 1 & - & - \\
\hline Saxifraga globulifera & - & - & - & - & + & - \\
\hline \multicolumn{7}{|l|}{ Compañeras } \\
\hline Bupleurum gibraltaricum & 1 & 2 & 1 & - & + & - \\
\hline Sedum dasyphyllum & - & + & 2 & + & - & - \\
\hline Sedum mисіzonia & - & - & 1 & - & 1 & V \\
\hline Rhamnus myrtifolius & + & 1 & - & - & - & - \\
\hline Stachys circinata & - & - & 1 & - & 1 & - \\
\hline Sedum sediforme & + & - & + & - & - & - \\
\hline Hedera helix & - & 1 & - & - & - & II \\
\hline Arisarum vulgare & - & - & - & - & - & II \\
\hline Crambe filiformis & - & - & - & - & + & - \\
\hline Festuca scariosa & - & 1 & - & - & - & - \\
\hline Galium album & - & - & 1 & - & - & - \\
\hline Geranium purpureum & - & - & - & - & - & II \\
\hline Phagnalon saxatile & - & - & - & - & + & - \\
\hline Phagnalon sordidum & - & - & + & - & - & - \\
\hline Sisymbrium laxiflorum & - & - & + & - & - & - \\
\hline
\end{tabular}

Localidades. 1. Málaga. Antequera. El Robledo. 30SUF7390. 2. Córdoba. Priego de Córdoba. Sierra Horconera. 30SUG8738. 3. Córdoba. Carcabuey. Umbría de la S ${ }^{\mathrm{a}}$ Gallinera. UG8440. 4. Córdoba. Rute. S de Rute. Cantera de Vichira. 30SUG8134. (invs. 3 y 4 proceden de Gómez Mercado et al., 2000). 5. Málaga. Cuevas de San Marcos. Sierra del Camorro. 30SUG7524. 6. Inventario sintético de la tabla correspondiente a Sarcocapno baetici-Centaureetum clementei (Asensi, 1976: 109) procedentes de Sierra de las Nieves (Málaga). 


\section{Series climatófilas}

Encinares

\section{Smilaco mauritanicae-Querceto rotundifoliae $S$}

Serie tingitano-onubo-algarviense, bética y mauritánica, termomediterránea, seco-subhúmeda de la encina (Quercus rotundifolia).

1a. Faciación basófila con encinas

\section{quercetoso rotundifoliae $s$}

La zonopotencialidad de las laderas sur de las sierras Torcalenses, hasta unos 900 metros de altitud, corresponde a encinares climatófilos y acebuchales edafoxerófilos. Los encinares, hoy día prácticamente extintos, de Smilaco-Quercetum rotundifoliae han sido sustituidos por su orla de Asparago -Rhamnetum oleoidis, espinar-lentiscar termófilo, visible sólo en reductos pedregosos no cultivados.

Donde el suelo se erosiona y en laderas de fuertes pendientes aparece el matorral de Odontito-Thymetum baetici. En zonas de cultivos marginales abandonados, la vegetación se recupera mediante una fase de retamar de Retamo-Genistetum speciosae. En esas mismas zonas, pero en laderas y antiguos bancales aún más térmicos aparecen cerrillares de Lotononido-Hyparrhenietum sinaicae. En las zonas sobrepastoreadas se desarrolla el cardal de Galactito-Cynaretum humilis.

\section{Paeonio coriaceae-Querceto rotundifoliae $S$}

Serie bética, basófila, mesomediterránea, seco-subhúmeda-húmeda de la encina (Quercus rotundifolia).

2a. Faciación típica quercetoso rotundifoliaes

2b. Faciación mesófila quercetoso fagineae s

2c. Faciación termófila con Pistacia lentiscus

La zonopotencialidad sobre las calizas y dolomías del piso mesomediterráneo Torcalense en el territorio es óptima para los encinares de Paeonio-Quercetum rotundifoliae. Estos encinares pueden mostrase puros (quercetosum rotundifoliae) en los biotopos más fríos y xéricos; como bosque mixto con quejigos (quercetosum fagineae) en biotopos con humedad compensada por umbrías, suelos profundos y zonas kársticas o ya en el tránsito al supramediterráneo; o por la faciación con Pistacia lentiscus en solanas y por debajo de aproximadamente $1000 \mathrm{~m}$. En el sotobosque, sobre todo en umbrías con sustrato rocoso, bajo las copas es frecuente un pastizal anual escionitrófilo de la comunidad de Cynosurus echinatus. La orla de estos bosques y etapa preforestal corresponde a un coscojar de Crataego-Quercetum cocciferae, aunque la faciación con quejigos puede tener una etapa preforestal de espinar caducifolio de SpartioRubetum ulmifolii y en zonas elevadas o navas con inversión térmica, se desarrollan espinares de la comunidad de Berberis hispanica $\mathrm{BC}$ de tránsito al supramediterráneo, siendo muy escasos y en zonas elevadas por encima de 1100 metros los escobonales de Genisto-Cytisetum reverchonii. Sobre suelos todavía profundos aunque algo erosionados por antiguos cultivos evolucionan retamales de Retamo-Genistetum speciosae.

Los matorrales de sustitución dependen de la posición geográfica de las sierras. En la zona más occidental (base del Torcal, Las Pedrizas, Sierra del Rey, Sierra de Alfarnate) y sobre suelos margosos aparece el Genisto speciosae-Ulicetum parviflorii, mientras que en la zona central (Zafarraya, Alcaucín) en laderas orientadas o abiertas hacia el sur, se desarrolla el matorral dolomitícola de Odontito-Thymetum baetici típico o lavanduletosum lanatae; en las planicies más interiores y orientales (Puerto de Los Alazores, Alhama) aparece el matorral de Thymo-Lavanduletum lanatae, que suele acompañarse en taludes y derrubios por vegetación glareícola magnesícola de la comunidad de Andryala ramossisima BC. Los lastonares de Helictotricho-Festucetum scariosae se encuentran en lapiaces con acúmulos de suelo. El pastizal anual pionero 
corresponde a una comunidad basófila de Trachynion distachyae $\mathrm{BC}$, frecuente en los claros de retamares no pastoreados. Bajo cubierta del propio encinar con quejigos, pero sobre rocas, hemos hallado el pastizal de Saxifrago-Hornungietum petraeae.

La vegetación exoserial está constituida por pastizales y comunidades rupícolas. En gran parte de estas unidades torcalenses son frecuentes majadales de la comunidad de Erodium primulaceum $\mathrm{BC}$ que ocupan porciones de suelos arcillosos en zonas karstificadas o, mucho más escasa, el Sedo-Crassuletum tillaeae poetosum bulbosae en litosuelos pedregosos. En zonas antropizadas y antiguamente cultivadas se encuentran pastizales ruderal-arvenses de Hordeion leporini y cardales de GalactitoCynaretum humilis, frecuentes en verano en zonas sobrepastoreadas La vegetación rupícola calcícola está constituida por el BiscutelloSaxifragetum reuteranae. Estos biotopos se enriquecen en las grandes oquedades nitrificadas y base de los extraplomos por el Stachydetum circinatae, mientras que en los paredones más extensos y verticales puede hallarse la escasa Linario-Centaureetum clementei.

\section{Berberido hispanicae-Querceto rotundifoliae $S$}

Serie bética, basófila, supramediterránea, seco-subhúmeda-húmeda de la encina (Quercus rotundifolia).

En las escasas sierras que sobrepasan los $1300 \mathrm{~m}$. de altitud, se desarrolla un incipiente piso bioclimático supramediterráneo, hoy día ocupado mayoritariamente por lastonares y piornales, ya que el biotopo corresponde a lapiaces rocosos de gran extensión. La zonopotencialidad climácica corresponde a encinares de Berberido-Quercetum rotundifoliae hoy día desaparecidos (criptoclímax). Sin embargo, se pueden encontrar representaciones de su orla espinosa caducifolia, correspondientes a la comunidad de Crataegus monogyna (a veces con Crataegus granatensis) e incluso, en zonas umbrías y navas con fuerte inversión térmica, a la comunidad de Berberis hispanica BC.

La mayor parte de estos territorios están ocupados por lastonares de HelictrotrichoFestucetum scariosae, que se desarrollan óptimamente en los acúmulos de suelo de las grietas de los lapiaces. En las zonas más elevadas y venteadas incluso se hallan pequeños fragmentos de piornales xeroacánticos de la comunidad de Ptilotrichum spinosum BC. Los litosuelos y pequeñas gleras calizas están colonizadas por el pastizal de VioloJonopsidietum prolongoi.

La vegetación exoserial aparece en los escasos roquedos verticales existentes y está compuesta por una comunidad rupícola que llega de modo finícola a estos territorios, el Saxifragetum camposii, así como, también finícola, la comunidad de caméfitos crasifolios Sedetum granatensis-acris en su variante termófila con Sedum mucizonia.

\section{Quejigares}

\section{Vinco difformis-Querceto fagineae $S$}

Serie antequerana (torcalense), calcícola, mesomediterránea, subhúmeda-húmeda del quejigo (Quercus faginea subsp. faginea).

4a. Faciación típica.

4b. Faciación edafohidrófila con Fraxinus

\section{angustifolia.}

En la unidad Torcalense, en el piso mesomediterráneo sobre calizas con ombrotipo húmedo y en tres tipos de biotopos básicos: suelos profundos en la base de sierras, laderas umbrías o, más rara vez, grandes lapiaces de zonas kársticas, se desarrollan quejigales de Vinco-Quercetum fagineae. En zonas con el suelo húmedo durante un mayor periodo, generalmente vaguadas, contactos con riberas o en la base de cantiles aparece la variante con Fraxinus angustifolia. Estos bosques presentan pastizales esciohumícolas de la comunidad de Cynosurus echinatus. Cuando el sotobosque 
umbroso del quejigal está sobrepastoreado se desarrolla un herbazal escionitrófilo vivaz de la comunidad de Smyrnium olusatrum. Cuando el bosque está bien conservado (muy rara vez) es muy buen indicador el herbazal esciohumícola de Clinopodio-Origanetum virentis. Estos bosques marcescentes están orlados por un espinar caducifolio de SpartioRubetum ulmifolii.

El matorral serial corresponde a Genisto speciosae-Ulicetum parviflorii, salvo en zonas elevadas donde aparece puntualmente un piornal de la comunidad de Ptilotrichum spinosum BC por encima de $1350 \mathrm{~m}$. En cultivos abandonados en zonas margosas la vegetación se recupera mediante el retamar de Retamo-Genistetum speciosae. También en esas zonas elevadas se puede hallar el nanopastizal calcícola de Violo demetriae-Jonopsidietum prolongoi. El pastizal anual más frecuente es de la comunidad de Brachypodium distachyon $\mathrm{BC}$.

Como vegetación exoserial, se desarrolla un pastizal escionitrófilo de oquedades calcáreas de Stachydetum circinatae así como la asociación Linario anticariaeSaxifragetum biternatae sobre calizas en grietas horizontales con orientación norte. En los roquedos con orientación norte y lapiaces profundos calizos es frecuente la comunidad pteridofítica de Polypodietum serrati. Los cardales de Galactito-Cynaretum humilis son típicos en verano en zonas sobrepastoreadas. Sobre arcillas contiguas a vaguadas o cursos de agua se desarrolla el prado hidrófilo de Elymo-Phalaridetum coerulescentis. En los bordes de los cultivos cerealísticos se puede observar la comunidad arvense de RidolphioCapnophylletum peregrini.

\section{Series edafoxerófilas}

Acebuchales

\section{Tamo communis-Oleeto sylvestris $\boldsymbol{S}$}

Serie iberomarroquí-atlántica, termomediterránea subhúmedo-húmeda del acebuche (Olea sylvestris)

5a. Faciación antequerana y malacitanoaxarquiense (bética) con Pistacia terebinthus.

Las laderas sur de la cordillera antequerana, de fuertes pendientes, muy rocosas, muy xéricas, térmicas y soleadas están caracterizadas por la presencia de acebuchales edafoxerófilos y arborescentes del Tamo-Oleetum sylvestris var. de Pistacia terebinthus (subrupícolas, basófilos). Esto ocurre en varias localidades del territorio, fundamentalmente base de la Sierra del Co, Puerto y Tajos del Sabar y Sierra del Rey. Normalmente el incendio y pastoreo de estos acebuchales los degrada a coscojarlentiscares de Asparago-Rhamnetum oleoides var. de Olea sylvestris

La vegetación exoserial en los roquedos calizos está constituida porel Linario-Centaureetum clementei o fragmentos muy termófilos del Biscutello-Saxifragetum reuteranae.

\section{Series edafohidrófilas}

Fresnedas

\section{Ficario ranunculoidis-Fraxineto angustifoliae salicetoso pedicellatae $s$}

Serie riparia iberomarroquí-atlántica del fresno (Fraxinus angustifolia)

6a. Faciación aljíbica y bética con Salix pedicellata.

Los escasos cursos de agua del territorio, generalmente intermitentes o con fuerte sequía estival, muestran zonopotencialidad para las fresnedas de Ficario-Fraxinetum angustifoliae salicetosum pedicellatae, hoy muy mermadas siendo frecuentes ejemplares aislados o pequeños grupos de Fraxinus angustifolia. La orla de este bosque ripario todavía es reconocible y corresponde a un espinar caducifolio de Spartio-Rubetum ulmifolii, mientras que en los propios lechos y pequeñas vegas deforestadas se encuentran juncales de Holoschoenetum vulgaris. En los 
suelos arcillosos que lindan con el curso de agua y por tanto con cierta hidromorfía, así como rodeando los juncales de esta serie se desarrolla un singular prado verticícola e hidrófilo de Elymo-Phalaridetum caerulescentis.

La zonopotencialidad para Fraxinus angustifolia creemos que puede también extenderse por las laderas arcillosas hidromorfas gran parte del año, así como en los territorios donde se desarrollan los quejigares más ombrófilos, en este caso como árbol acompañante.

Olmedas

\section{Opopanaco chironii-Ulmeto minoris $\boldsymbol{S}$}

Serie riparia ibérica del olmo (Ulmus minor)

En el sector Torcalense son frecuentes las surgencias en el contacto entre las calizas y las arcillas. Estos biotopos están generalmente colonizados por las olmedas de OpopanacoUlmetum minoris o su orla, un zarzal de Spartio-Rubetum ulmifolii, pudiéndose observar juncales de Holoschoenetum vulgaris bordeando el propio curso de agua.

Adelfares

\section{Rubo ulmifolii-Nerieto oleandri $S$}

Serie riparia ibérica, termomesomediterránea inferior de la adelfa (Nerium oleander).

La zonopotencialidad de los cursos de agua intermitentes del piso termomediterráneo en la cordillera antequerana corresponde a adelfares de Rubo-Nerietum oleandri, escasos ya que casi toda esta cordillera pertenece al piso meso y supramediterráneo y además, debido a la karstificación, es una zona muy pobre en cursos de agua superficiales. Dichos adelfares pueden alternar con zarzales de SpartioRubetum ulmifolii. La desaparición de esta asociación conlleva el desarrollo de juncales de Holoschoenetum vulgaris.

\section{Complejos edafogénicos}

Karsticícolas

\section{Comunidad de Acer monspessulanum y} Pistacia terebinthus

Los acerales ocupan biotopos muy definidos, siempre ligados a la geomorfología kárstica: lapiaces, canchales, base de cantiles, casi siempre en la umbría de las sierras. Debido a sus apetencias subrupícolas, el sotobosque presenta un pastizal escionitrófilo de la comunidad de Geranium lucidum BC, pudiendo estar presentes especies de la orla de la comunidad de Crataegus monogyna, con Prunus mahaleb y Rhamnus saxatilis en este caso. En la misma base de los cantiles se desarrolla el herbazal escionitrófilo de Smyrnium olusatrum. Las etapas seriales de matorral son muy fragmentarias, debido a la geomorfología kárstica, aunque existen especies del Genisto-Ulicetum parviflorii, sobre todo Ulex parviflorus. El pastizal más frecuente es el majadal de la comunidad de Erodium primulaceum $\mathrm{BC}$ en las pequeñas navas.

La vegetación exoserial rupícola corresponde al Biscutello-Saxifragetum reuteranae en zonas medias y en su variante de Erodium cheilanthifolium en zonas más elevadas o umbrías; en oquedades nitrificadas aparece el Stachydetum circinatae.

\section{Dulceacuícolas}

\section{Comunidad de Juncus inflexus \\ 11. Callitricho stagnalis-Ranunculetum saniculifolii}

\section{Ranunculetum tricophylli}

Complejos restringidos a los fragmentarios biotopos con agua edáfica o estancada todo el año del Poljé de Zafarraya, donde se presentan la comunidad de Juncus inflexus con Eleocharis palustris en zonas encharcadizas y Callitricho stagnalis-Ranunculetum saniculifolii en pequeñas charcas semi-naturales. En el río 


\begin{tabular}{|c|c|c|c|}
\hline Litología & Piso bioclimático & Ombrotipo & Zonopotencialidad climácica \\
\hline Calizas & $\begin{array}{c}\text { termo al } \\
\text { supramediterráneo }\end{array}$ & $\begin{array}{l}\text { subhúmedo- } \\
\text { húmedo }\end{array}$ & $\begin{array}{c}\text { encinares, quejigares, } \\
\text { acebuchales } \\
\text { (Quercus rotundifolia, Quercus } \\
\text { faginea, Olea sylvestris) }\end{array}$ \\
\hline Dolomías & mesomediterráneo & $\begin{array}{l}\text { subhúmedo- } \\
\text { húmedo }\end{array}$ & $\begin{array}{c}\text { encinares } \\
\text { (Quercus rotundifolia) }\end{array}$ \\
\hline Arcillas, margas & $\begin{array}{c}\text { termo y } \\
\text { mesomediterráneo }\end{array}$ & $\begin{array}{l}\text { subhúmedo- } \\
\text { húmedo }\end{array}$ & $\begin{array}{c}\text { quejigales y encinares } \\
\text { (Quercus faginea, Quercus } \\
\text { rotundifolia) }\end{array}$ \\
\hline Poljés (navas) & mesomediterráneo & $\begin{array}{l}\text { subhúmedo- } \\
\text { húmedo }\end{array}$ & $\begin{array}{c}\text { quejigales (Quercus faginea) y } \\
\text { vegetación hidrófita }\end{array}$ \\
\hline Ríos & $\begin{array}{c}\text { termo y } \\
\text { mesomediterráneo }\end{array}$ & subhúmedo & $\begin{array}{l}\text { fresnedas, olmedas } \\
\text { (Fraxinus angustifolia, } \\
\text { Ulmus minor) }\end{array}$ \\
\hline
\end{tabular}

Tabla 11. Formaciones climácicas boscosas y arbustivas del territorio según la zonopotencialidad litológica y bioclimática. Forests and shrub vegetation in the study area according to zono-potential lithology and bioclimatology.

Sabar (Alfarnatejo) aparece el Ranunculetum tricophylli en remansos no muy someros.

\section{Zonopotencialidad fitogeográfica (Paisaje Vegetal)}

La tabla 11 muestra las formaciones y especies climácicas en función de su respectiva zonopotencialidad bioclimática y litológica.

\section{ESQUEMA SINTAXONÓMICO}

Se presenta a continuación la clasificación fitosociológica de la vegetación del área de estudio. Cada asociación, subasociación, comunidad (BC) y variante se acompañan de una breve descripción con datos fisionómicos, ecológicos y/o corológicos.

\section{Vegetación boscosa y orlas arbustivas siempreverdes y aciculifolias}

QUERCETEA ILICIS Br.-B1. 1947

+ Quercetalia ilicis Br.-Bl. ex Molinier 1934 em. Rivas-Martínez 1975

* Querco-Oleion sylvestris Barbero, Quèzel \& Rivas Martínez 1981

1.Smilaco mauritanicae-Quercetum rotundifoliae Barbero, Quèzel \& Rivas Martínez 1981 quercetosum rotundifoliae [Encinares termomediterráneos]

2. Tamo communis-Oleetum sylvestris Benabid ex Pérez Latorre, Galán de Mera, Deil \& Cabezudo 1996 [Acebuchales]

* Quercion broteroi Br.-Bl., P. Silva \& Rozeira 1956 corr. Ladero 1974 em. Rivas-Martínez 1975

** Paeonio broteroi-Quercenion rotundifoliae Rivas Martínez in Rivas Martínez, Costa e Izco 1986

3. Paeonio coriaceae-Quercetum rotundifoliae Rivas Martínez 1975

quercetosum rotundifoliae [Encinares mesomediterráneos basófilos]

var. de Pistacia lentiscus [Encinares termófilos] quercetosum fagineae Rivas Martínez 1964 em. Rivas Goday \& Rivas Martínez 1971 [Encinares con quejigos]

+ Pistacio lentisci-Rhamnetalia alaterni RivasMartínez 1975

* Rhamno lycioidis-Quercion cocciferae Rivas Goday ex Rivas Martínez 1975

4. Crataego monogynae-Quercetum cocciferae Martínez Parras, Peinado \& Alcaraz 1985 [Coscojares mesomediterráneos basófilos]

II. Vegetación potencial caducifolia y aciculifolia y sus orlas arbustivas. Vegetación riparia.

QUERCO-FAGETEA Br.-Bl. \& Vlieger in Vlieger 1937 
+ Quercetalia pubescenti-petraeae Klika 1933

* Aceri granatensis-Quercion fagineae (Rivas Goday, Rigual \& Rivas Martínez in Rivas Goday et al. 1960) Rivas Martínez 1987

5. Vinco difformis-Quercetum fagineae Pérez Latorre \& Cabezudo ass. nova quercetosum fagineae [Quejigales mesomediterráneos]

var. de Fraxinus angustifolia [Variante verticícolo-hidrófila con fresnos]

6. Comunidad de Acer monspessulanum y Pistacia terebinthus [Aceral con terebintos]

+ Populetalia albae Br.-Bl. ex Tchou 1948

* Populion albae Br.-Bl. ex Tchou 1948

** Fraxino angustifoliae-Ulmenion minoris Rivas Martínez 1975

7. Ficario ranunculoidis-Fraxinetum angustifoliae Rivas-Martínez \& Costa in RivasMartínez, Costa, Castroviejo y Valdés 1980

salicetosum pedicellatae Díez Garretas, Cuenca \& Asensi 1986 [Fresnedas con sauces arbustivos]

8. Opopanaco chironii-Ulmetum minoris Bellot \& Ron in Bellot, Ron \& Carballal 1979 [Olmedas]

+ Prunetalia spinosae Tüxen 1952

* Lonicero arboreae-Berberidion hispanicae O.

Bolòs 1954

9. Comunidad de Berberis hispanica [Espinares con agracejo]

* Pruno-Rubion ulmifolii O. Bolòs 1954

** Pruno-Rubenion ulmifolii

10. Lonicero hispanicae-Rubetum ulmifolii Rivas Martínez, Costa, Castroviejo \& Valdés Bermejo 1980 [Zarzal con madreselvas]

11. Spartiojuncei-Rubetum ulmifolii Pérez Latorre \& Cabezudo 2008 [Zarzal con gayumbas]

** Rosenion carioto-pouzinii Arnaiz ex Loidi 1989

12. Comunidad de Crataegus monogyna sensu Pérez Latorre et al. 1998 [Espinar-zarzal mesomediterráneo basófilo]

NERIO-TAMARICETEA Br.-Bl. \& O. Bolòs 1958

+ Tamaricetalia africanae Br.-B1. \& O. Bolòs 1958 em. Izco, Fernández-González \& Molina 1984

* Rubo ulmifolii-Nerion oleandri O. Bolòs 1985

13. Rubo ulmifolii-Nerietum oleandri $\mathrm{O}$. Bolòs 1956 [Adelfares]

III. Orlas genistoides, matorrales y tomillares.

CYTISETEA SCOPARIO-STRIATII Rivas Martínez
1974

+ Cytisetalia scopario-striatii Rivas Martínez 1974

* Retamion sphaerocarpae Rivas-Martínez 1981

14. Retamo sphaerocarpae-Genistetum speciosae Rivas Martínez ex Martínez Parras, Peinado y Alcaraz, 1983 [Retamares basófilos]

* Adenocarpion decorticantis (Rivas-Martínez \& F. Valle ex F. Valle 1985) Rivas-Martínez, FernándezGonzález \& Loidi 1999

15. Genisto speciosae-Cytisetum reverchonii F. Valle 1987 [Escobonales basófilos]

CISTO-MICROMERIETEA JULIANAE Oberdorfer 1954

+ Rosmarinetalia Br.-B1. 1931

* Saturejo micranthae-Thymbrion capitati RivasGoday \& Rivas-Martínez 1969

16. Odontito purpureae-Thymetum baetici Esteve \& López Guadalupe 1977

thymetosum baetici [Matorrales dolomitícolas termófilos]

lavanduletosum lanatae Pérez Latorre \& Cabezudo in Pérez Latorre, D. Navas, O. Gavira, G. Caballero \& Cabezudo 2004 [Matorrales dolomitícolas mesomediterráneos termófilos]

17. Thymo gracile-Lavanduletum lanatae Pérez Raya \& Molero Mesa 1988 [Matorrales dolomitícolas mesomediterráneos continentales] 18. Genisto speciosae-Ulicetum parviflorii Rivas Goday \& Rivas-Martínez ex Pérez Latorre y Cabezudo nom. inv. propos. [Matorrales calcícolas]

+ Erinacetalia anthyllidis Quézel 1951

* Xeroacantho-Erinaceion anthyllidis (Quézel 1953)

O. Bolòs 1967

19. Comunidad de Ptilotrichum spinosum BC [Piornal xeroacántico]

\section{Vegetación de pastizales y prados}

HELIANTHEMETEA ANNUAE (Br.-Bl. in Br.-Bl. et al. 1952) Rivas Goday \& Rivas-Martínez 1963 em. Rivas-Martínez 1978

+ Trachynietalia distachyae Rivas-Martínez 1978

* Trachynion distachyae Rivas-Martínez 1978 20. Comunidad de Brachypodium dystachion BC [Pastizal anual basófilo de suelos arcillosos] 21. Sedo caespitosii-Crassuletum tillaeae Rivas Goday 1958

poetosum bulbosae Pérez Latorre \& Cabezudo 
2008 [Pastizal crasifolio pastoreado]

22. Violo demetriae-Jonopsidietum prolongoi Asensi, Díez-Garretas \& Esteve 1979 [Pastizal anual fugaz de litosuelos calizos]

23. Saxifrago tridactylitae-Hornungietum petraeae Izco 1974 [Pastizal anual subrupícola]

\section{LYGEO SPARTI-STIPETEA TENACISSIMAE Rivas-} Martínez 1978

+ Lygeo sparti-Stipetalia tenacissimae Br-B1. \& O. Bolòs 1958 em. Rivas-Martínez 1978

* Festucion scariosae Martínez Parras, Peinado \& Alcaraz 1983

24. Helictotricho filifolii-Festucetum scariosae Martínez Parras, Peinado \& Alcaraz 1983 [Lastonares]

* Stipion tenacissimae Rivas-Martínez 1978

25. Thymo gracilis-Stipetum tenacissimae Pérez Raya \& Molero 1988 [Espartales]

+ Hyparrhenietalia hirtae Rivas-Martínez 1978

* Hyparrhenion hirtae Br.-Bl., P. Silva \& Rozeira 1956

26. Lotononido lupinifoliae-Hyparrhenietum sinaicae Díez Garretas \& Asensi 1999 [Cerrillares]

TRIFOLIO-GERANIETEA SANGUINEI (Th. Müller 1961) Rivas-Martínez \& Cantó 1987

+ Origanetalia vulgaris Th. Müller 1961 (1962)

* Origanion virentis Rivas-Martínez \& O. Bolòs in Rivas-Martínez \& col. 1984

27. Clinopodio arundani-Origanetum virentis Rivas Martínez in Rivas Martínez et al. 1984 [Herbazal esciohumícola]

POETEA BULBOSAE Rivas Goday \& RivasMartínez in Rivas-Martínez 1978

+ Poetalia bulbosae Rivas Goday \& Rivas-Martínez in Rivas Goday \& Ladero 1970

* Plantaginion serrariae Galán de Mera, Morales \& Vicente Orellana 2000

28. Comunidad de Erodium primulaceum BC [Majadal basófilo]

SEDO-SCLERANTHETEA Br. - B1. 1955 em. Th. Muller 1961

+ Alysso-Sedetalia Moravec 1967

* Alysso-Sedion albi Oberdorfer \& Müller in Müller 1961

29. Sedetum granatensis-acris Pérez Latorre \&
Cabezudo in Pérez Latorre, D. Navas, O. Gavira, G. Caballero \& Cabezudo 2004 var. de Sedum mucizonia [Pastizal crasifolio, variante termófila]

\section{Vegetación nitrófila, viaria y arvense}

ARTEMISIETEA VULGARIS Lohmeyer, Preising \& Tüxen in Tüxen 1950 ampl. Rivas-Martínez, Báscones, T.E. Díaz, Fernández González \& Loidi 1991

+ Carthametalia lanati Brullo in Brullo \& Marceno 1985

* Onopordion castellani Br.-Bl. \& O. Bolòs 1958 corr. Rivas-. Martínez, T.E. Díaz, FernándezGonzález, Izco, Loidi, Lousã \& Penas 2002

30. Galactito tomentosae-Cynaretum humilis Rivas Goday 1964 nom. inv. propos. [Cardales estivales]

* Sylibion mariani Rivas Martínez 1987

31. Carduo bourgaeani-Sylibetum mariani Rivas Martínez ex Rivas Martínez, Costa \& Loidi 1992 [Cardales primaverales]

+ Ononidetalia ramosissimae Galán de Mera, Sánchez García \& Vicente Orellana 1997

* Bromo-Oryzopsion miliaceae O. Bolòs 1970

32. Inulo viscosae-Oryzopsietum milliaceae (A. \& O. Bolòs 1950) O. Bolòs 1957 [Altabacares]

GALIO-URTICETEA Passarge ex Kopecky 1969

+ Galio aparines-Alliarietalia petiolatae Görs \& Muller 1969 em. Rivas Martínez 1987

* Galio-Alliarion petiolatae Oberdorfer \& Lohmeyer in Oberdorfer, Görs, Korneck, Lohmeyer, Müller, Philippi \& Seibert 1967

33. Com. de Smyrnium olusatrum BC [Herbazal escionitrófilo]

STELLARIETEA MEDIAE R.Tx., Lohmeyer \& Preising in R. Tx. 1950 em. Rivas-Martínez 1977

+ Centaureetalia cyani Tüxen ex Von Rochow 1951

* Ridolphion segeti Nègre ex Rivas Martínez, Fernández González \& Loidi 1999

34. Ridolphio segeti-Capnophylletum peregrini Guinochet 1978 [Pastizales arvenses de cereal]

+ Geranio purpurei-Cardaminetalia hirsutae Brullo in Brullo \& Marceno 1985

* Geranio pusilli-Anthriscion caucalidis Rivas Martínez 1978 
35. Comunidad de Cynosurus echinatus [Pastizal anual escionitrófilo de sotobosques]

36. Comunidad de Geranium lucidum BC sensu Pérez Latorre et al., 1998 [Pastizal anual escionitrófilo subrupícola]

+ Sisymbrietalia officinalis J. Tüxen in Lohmeyer et al. 1962 em. Rivas-Martínez, Báscones, T.E. Díaz, Fernández-González \& Loidi 1991

* Hordeion leporini Br.-B1. in Br.-B1., Gajewski, Wraber \& Walas 1936

37. Comunidad de Plantago lagopus BC [Herbazal de cultivos abandonados]

\section{Vegetación rupícola y subrupícola (taludes, gleras)}

ANOMODONTO-POLYPODIETEA Rivas-Martínez 1975

+ Anomodonto-Polypodietalia O. Bolòs \& Vives in O. Bolòs 1957

* Polypodion serrulati (cambrici) Br.-Bl. in Br.-Bl. et al. 1952 corr.

38. Polypodietum serrati Br.-Bl. in Br.-Bl., Roussine \& Nègre 1952 [Comunidad rupícola pteridofítica umbrófila]

ASPLENIETEA TRICHOMANIS (Br.-Bl. in Meier \& Br.-B1. 1934) Oberdorfer 1977

+ Asplenietalia petrarchae (glandulosi) Br.-Bl. in Meier \& Br.-Bl. 1934

++ Tinguarrenalia siculae (Daumas, Quèzel \& Santa 1952) Galán de Mera in Pérez Latorre, Galán de Mera, Deil \& Cabezudo 1996

* Campanulion velutinae Martínez Parras \& Peinado 1990

** Campanulenion velutinae

39. Biscutello frutescentis-Saxifragetum reuteranae Socorro \& Marín Calderón 1983 saxifragetosum reuteranae [Vegetación rupícola termófila]

var. de Hippocrepis rupestris [Variante heliófila]

var. de Erodium cheilanthifolium [Variante orófila]

40. Linario anticariae-Saxifragetum biternatae Esteve \& López Guadalupe 1973 [Vegetación rupícola mesófila]

** Centaureenion clementei Pérez Latorre \& Cabezudo suball. nova

\section{Linario anticariae-Centaureetum clementei}

Pérez Latorre \& Cabezudo ass. nova [Vegetación rupícola camefítica]

+ Potentilletalia caulescentis Br.-B1. in Br.-B1. \& Jenny 1926

* Saxifragion camposii Cuatrecasas ex Quézel 1953

42. Saxifragetum camposii Cuatrecasas ex Martínez Parras \& Peinado Lorca 1990 [Vegetación rupícola supramediterránea]

+ Parietarietalia judaicae Rivas-Martínez in Rivas Goday 1964

* Parietario-Galion murale Rivas Martínez ex Rivas Goday 1964

43. Stachydetum circinatae Fdez. Casas 1972 [Comunidad de oquedades nitrificadas]

+ Phagnalo-Rumicetalia indurati Rivas Goday \& Esteve 1972

* Andryalo-Crambion filiformis (Rivas Goday \& esteve 1972) Rivas Martínez, Izco \& Costa 1973

44. Comunidad de Andryala ramosissima BC [Comunidad glareícola-magnesícola]

VII. Vegetación de suelos húmedos, acuática, helofítica y anfibia

MOLINIO-ARRHENATHERETEA R. Tx. 1937

+ Holoschoenetalia Br.-Bl. (1931) 1947

* Molinio-Holoschoenion Br-Bl. (1931) 1947

45. Holoschoenetum vulgaris $\mathrm{Br}$.-Bl. ex Tchou 1948 [Juncales]

+ Plantaginetalia majoris R. Tx. \& Preising in R.

Tx. 1950 em Rivas-Martínez 1987

* Mentho-Juncion inflexi De Foucault 1984

46. Comunidad de Juncus inflexus [Juncales nitrófilos]

+ Phalaridetalia coerulescentis A. Galán, Deil, Haug \& Vicente Orellana 1997

* Gaudinio fragilis-Hordeion bulbosi A. Galán, Deil,

Haug \& Vicente Orellana 1997

47. Elymo repentis-Phalaridetum coerulescentis Pérez Latorre, Galán \& Cabezudo in Pérez Latorre, P. Navas, D. Navas, Y. Gil \& Cabezudo 1998 [Herbazal verticícola hidrófilo]

POTAMETEA Klika in Klika \& Novák 1941

+ Potametalia W. Koch 1926

* Ranunculion aquatilis Passarge 1964

48. Callitricho stagnalis-Ranunculetum saniculifolii Galán in A. V. Pérez, Galán, P. Navas, D. Navas, Y. Gil \& Cabezudo 1999 [Comunidad 
de hidrófitos en aguas someras]

* Ranunculion fluitantis Neuhaüsel 1959

49. Ranunculetum tricophylli Melendo, Cano \& Valle 2003 [Comunidad de hidrófitos en remansos de arroyos]

AGRADECIMIENTOS. Al Dr. A. Galán de Mera por sus sugerencias y comentarios al manuscrito.

\section{BIBLIOGRAFÍA}

ASENSI, A. -1976- Flora y Vegetación de las áreas ocupadas por el Abies pinsapo Boiss. Tesis Doctoral. Universidad de Granada.

ASENSI A., B. DÍEZ GARRETAS y J. M. NIETO -2005- Torcal de Antequera-Desfiladero de Los Gaitanes. Guía Geobotánica. XX Jornadas de Fitosociología. Málaga. 87 pp.

BRAUN-BLANQUET, J. -1979-. Fitosociología. Ed. Blume. Madrid.

CABEZUDO, B., J. MOLERO MESAy A. V. PÉREZ LATORRE. -1998-. Vegetación de Andalucía. En: Carmen Rodríguez Hiraldo (coord.). La Flora (tomo 3). Naturaleza de Andalucía (obra completa). Ediciones Giralda. Sevilla.

CANO, E., C. PINTO-GOMES, F. VALLE, J. A. TORRES, A. GARCÍA FUENTES, C. SALAZAR, M. MELENDO y S. MENDES -2001- Primera aproximación al conocimiento de los quejigares del sur de la Península Ibérica (Portugal y España). Quercetea 3: 175-182.

CASTROVIEJO, S. (coord.) -1986-2008- Flora Iberica. Real Jardín Botánico. CSIC. Madrid.

CEBALLOS, L. y C. VICIOSO. -1933-Estudio sobre la vegetación y flora forestal de la provincia de Málaga. Inst. Forestal de Invest. y Exp. Madrid.

DIERSCHKE, H. -1993- Grundlagen und Methoden der Planzensoziologie. Ulmer. Stuttgart.

FOCAULT,B.-1981-Réflexionssul'apprauvrissement des syntaxons aux limites chorologiques des unités phytosociologiques supérieurs et quelques unes de leurs consequences. Lazaroa 3: $75-100$.

GALÁN DE MERA, A., A. V. PÉREZ LATORRE y J. A. VICENTE ORELLANA-2003- Relaciones fitogeográficas entre el suroccidente de la Península Ibérica y el noroeste de África. Una propuesta de sectorización. Lagascalia 23: 27-52.

GAVIRA, O. -2006- Análisis de un corredor florístico entre los Parques Naturales Sierra de Grazalema y Sierra de las Nieves (Cádiz y Málaga, España). Anales de Biología 28: 45-72.

GEHÚ, J. M. y S. RIVAS-MARTÍNEZ -1981Notions fondamentales de Phytosociologie. Syntaxonomie. Berichte der Internationalen Symposien der Internationalen Vereinigung für Vegetationskunde.

GÓMEZ MERCADO, F., J. F. MOTA, J. PEÑAS, J. CABELLO y F. VALLE -2000- Vegetación del Parque Natural Sierra Subbética (Córdoba). En: J. M. Moreira (ed.). Reconocimiento Biofísico de Espacios Naturales Protegidos: Parque Natural de Las Sierras Subbéticas, pp. 197-288. Consejería de Medio Ambiente, Junta de Andalucía.

GUERRA, J. -1982- Catálogo de la flora vascular de la Sierra del Torcal de Antequera (Málaga). Inst. Est. Almerienses. Homenaje almeriense al botánico Rufino Sagredo: 105-120.

GUERRA, J., E. SALVO, J. M. NIETO y B. CABEZUDO -1988-Espacios de interés natural de la provincia de Málaga: II. Parque natural del Torcal de Antequera. Jábega 60: 75-80.

KOPECKÝ, K., J. DOSTALEK \& T. FRANTIK -1995- Biological invasion on an oceanic island mountain: do alien plant species have wider ecological ranges than native species? J. Veg. Sci. 6: 667-674.

NIETO CALDERA, J. M., A. V. PÉREZ LATORRE y B. CABEZUDO -1991-. Biogeografía y series de vegetación de la provincia de Málaga (España). Acta Bot. Malacitana 16(2): 417-436.

NIETO J. M., B. CABEZUDO, A. V. PÉREZ LATORRE, D. NAVAS, P. NAVAS y Y. GIL -1998- Apuntes para el estudio del paisaje vegetal de la provincia de Málaga. En: Rebollo M., F. Serrano, J. M. Nieto y B. Cabezudo (Eds.). Itinerarios por Espacios Naturales de la provincia de Málaga. Servicio de publicaciones. Universidad de Málaga.

PÉREZ LATORRE A. V., P. NAVAS, D. NAVAS, Y. GIL y B. CABEZUDO -1998-. Datos sobre la Flora y Vegetación de la Serranía de Ronda (Málaga, España). Acta Bot. Malacitana 23:149191.

PÉREZ LATORRE A. V. y B. CABEZUDO -2002- 
La flora y el paisaje vegetal de la provincia de Málaga: importancia y conservación. Jábega 90: 25-39.

PÉREZ LATORREA. V., D. NAVAS FERNÁNDEZ, O. GAVIRA, G. CABALLEROy B. CABEZUDO -2004- Vegetación del P. N. de las Sierras Tejeda, Almijara y Alhama. Acta Bot. Malacitana 29: 117-190.

PÉREZ LATORRE A. V., G. CABALLERO, F. CASIMIRO-SORIGUER, O. GAVIRA y B. CABEZUDO -2008- Vegetación del sector Malacitano-Axarquiense (comarca de la Axarquía, Montes de Málaga y Corredor de Colmenar). Málaga (España). Acta Bot. Malacitana 33: 215-270.

RIVAS GODAY, S. y S. RIVAS MARTÍNEZ -1968Matorrales y Tomillares de la Península Ibérica comprendidos en la clase Ononido-Rosmarinetea. Anales Inst. Bot. Cavanilles 25:5-180.

RIVAS MARTINEZ, S. -1987- Memoria y mapa de las series de vegetación de España (1: 400.000). ICONA. Madrid.

RIVAS MARTÍNEZ, S. -1988- Bioclimatología, biogeografía y series de vegetación de Andalucía Occidental. Lagascalia 15 (extra): 91-119.

RIVAS MARTÍNEZ S., A. ASENSI, B. DÍEZ GARRETAS y J. MOLERO -1997Biogeographical synthesis of Andalusia (southern Spain). Journal of Biogeography 24: 915-928.

RIVAS MARTÍNEZ, S., F. FERNÁNDEZ GONZÁLEZ, J. LOIDI, M. LOUSAy A. PENAS
-2001- Syntaxonomical check-list of vascular plant communities of Spain and Portugal to association level. Itinera Geobotanica 14: 5341.

RIVAS MARTÍNEZ, S., T. E. DÍAZ, F. FERNÁNDEZ GONZÁLEZ, J. IZCO, J. LOIDI y A. PENAS -2002-Vascular plant communities of Spain and Portugal. Itinera Geobotanica 15(1): 5-432.

SCHUHWERK, F. -1990- Relikte und Endemiten in Pflanzengesellschaften Bayerns- eine vorlaufige Ubersicht. Ber. Bayer. Bot. Ges. 61: 303-323.

SOCORRO ABREU, O. y G. MARÍN CALDERÓN -1983- Notas fitosociológicas béticas, I. Anales Jard. Bot. Madrid 39 (2): 515-518.

VALDÉS, B., M. REJDALI, A. ACHHAL, J. L., JURY y J. M. MONSERRAT (Eds.) -2002Catalogue des Plantes Vasculaires du Nord du Maroc, incluant des clés d'identification. Biblioteca de Ciencias. CSIC. Madrid.

VALDÉS, B., S. TALAVERA y E. F. GALIANO (eds.) -1987- Flora vascular de Andalucía Occidental. Ed. Ketres. Barcelona.

VIGO, J. -1998- Some reflections on geobotany and vegetation mapping. Acta Bot. Barc. 45: 535-566.

WEBER, H. E., J. MORAVEC \& J. P. THEURILLAT -2000- International Code of Phytosociological Nomenclature. J. Veg. Sci. 11: 739-768.

YUS RAMOS, R., M. A. TORRES y F. BOTELLA -2007-Por la Dorsal Bética. CEDER Axarquía. Diputación de Málaga, 299 pp. 